\title{
Ueber den Einfluss der Mydriatica und Myotica auf den intraocularen Druck unter physiologischen Verhältnissen.
}

\author{
Von \\ Dr. Friedrich Stocker \\ aus Luzern.
}

v. Graefe gebührt das grosse Verdienst, die sehr versehiedenen klinischen Bilder, die wir mit "Glaucom" bezeichnen, und die fast ausnahmslos zur Erblindung des erkrankten Auges führten, durch Zusammenfassung unter einen gemeinsamen pathogenetischen Begriff unserm Verständniss näher gerückt und für eine grosse Zahl der Fälle der Therapie und der Heilung zugänglich gemacht zu haben.

Während man im Anfang dieses Jahrhunderts im Glaucom eine Erkrankung des Nervus opticus, der Retina oder der Chorioidea wahrzunehmen glaubte, definirte v. Graefe*) diese Krankheit als eine Vermehrung der intraocularen Spannung mit Rückwirkung auf die Functionen des Sehnerven, resp. der Netzhaut. Die Iridectomie, welche v. Graefe gegen diese Cardinalsymptome des Glancoms

*) Graefe's Archiv fitr Ophthalmologie Bd. I. pag. 279. 
richtete, zeigte sich in einer grossen Zahl von Füllen als souverăn und befestigte daher die v. Graefe'sche Auffassung der Glaucomlehre, die eine Zeit lang von allen Ophthalmologen getheilt wurde. Der Dienst, den $r$. Graefe der Menschheit mit der Glaucom-Iridectomie erwiesen, wird nicht weniger unsterblich bleiben, wenn auch die Anschauungen, die er über das Wesen des Glaucoms seiner Mitwelt lehrte, nach and nach der Modificirung und Umwandlung unterliegen werden.

Wie jeder Fortschritt in Etwas durch die Bewegung in den Extremen sich charalterisirt, so ist der entschiedenste Gegner der v. Graefe'schen Glaucomlehre, Mauthner*), dahin gekommen, dem erhöhten intraoculären Druck jede Bedeutung für die Krankheit abzusprechen. Wenn die klinische Erfahrung auch zur Gentige lehrt, dass die erhohte intraoculare Spamung nicht der einzig wirkende fatale Faktor beim Glaucom sein kann, so sprechen doch wieder Thatsachen dafür, dass im Circulus vitiosus von Ursache und Wirkung bei dem zur Stunde noch keineswegs genügsam aufgeklärten Krankheitsprocess der erhöhte intraoculäre Druck eine Bedeutung beansprucht.

Hatte man auch schon vor Graefe gewusst, dass der Bulbus als eine mit Flüssigkeit gefüllte Blase unter einem gewissen Druok stehe, so gab man sich mit dieser physiologischen Thatsache nicht weiter ab, indem man eben ihre pathologische Bedeutung noch nicht kannte. Exst nachdem das Glaucom durch Graefe als Drucksteigerung definirt worden, trat die Abschätzung oder Messung der Augenspannung in den Vordergrund der Thätigkeit eines jeden Ophthalmologen.

Es haben sich mit der Zeit namentlich zwei Methoden gezeigt, wodurch der Binnendruck des Auges objectiv zur

*) Archiv f. Augen- u. Ohrenheilk. Bd. VII. Mauthner: Vorträge aus dem Gesammtgebiet der Augenheilkunde Heft IX, $\mathrm{X}$ u. XI. 
Ueber den Einfluss der Mydriatica und Myotica etc. 107

Anschauung gebracht werden sollte, die Tonometrie und die Manometrie.

Das allererste Verfahren war die Prüfung der Augenspannung vermittelst der aufgelegten Zeigefinger beider Hände; ja diese Art der Palpation wird hent zu Tage noch von darin Geübten mit grossem Erfolg betrieben, natürlich nur, wo es sich um relative Werthe, Druckunterschiede zwischen zwei Augen, handelt.

Die Tonometer, deren es verschiedene Arten giebt: z. B. von Snellen, Dor, Weber, sind auf Quecksilbermillimeter graduirt, respective die Tonometergrade können auf $\mathrm{Hg}$-Millimeter reducirt werden.

Die Druckhöhen liegen zwisehen 20 bis 50 Millimeter Quecksilber. Leber*) bezeichnet die Ableitung der Druckhöhen aus den Tonometergraden als eine sehr unsichere, da E. Pflüger mit dem verbesserten Dor'schen Instrument bei einer Zunahme des Augendruckes ron 20 bis auf 50 Millimeter ein Steigen der mittleren Tonometerwerthe nur von 20 bis $24,7^{\circ}$ gefunden, während bei Dor einem Steigen von 20 bis $30 \mathrm{Hg}$-Millimeter eine ungefäbr gleiche Zunahme der mittleren Tonometerwerthe $\left(20-24^{3} / 4^{0}\right)$ entsprach.

Was die manometrisehe Bestimmung des Augendruckes betrifft, so wollen wir uns deren Entwickelung etwas näher besehen, da wir bei unserer Arbeit von dieser Methode ausgegangen sind.

Grünhagen**), Adamük***) und Wegner $\dagger$ ) haben so ziemlich zur gleichen Zeit, im Jahre 1866, die manometrische Untersuchungsmethode zur Prüfung des Augen-

*) A. Graefe und Saemisch: Handbuch der gesammten Angenheilkunde. II. 369 .

*) "Ueber den intraoculären Druck". Berliner klin. Wochenschrift No. 24 and Zeitschrift fir ration. Medizin 3, XXVIII.

***) Centralblatt $f$. mediz. Wissenschaften No. 36 .

$\dagger$ ) „Experimenteller Beitrag z. Lehre rom Glaucom." Archiv für Ophthalm. XII. II, I. 
druckes eingeführt. Ersterer benutzte eine Stichcanüle, welche durch eine seitliche Oeffnung mit einem einfachen $\mathrm{Hg}$-Manometer communicirte. $\mathrm{Er}$ stach in die vordere Augenkammer. Der Raum zwischen Kammerwasser und dem Quecksilber des Manometers wurde mit Oel gefullt, damit eine Mischung der Flüssigkeiten unmöglich werde. Bevor der Einstich gemacht wurde, erzeugte man bis zu 20 Hg-Millimeter Ueberdruek im Manometer durch Aufgiessen von Quecksilber in dasselbe. Ein Ueberdruck von 15 bis 20 Millimetern sollte den gefundenen mittleren Augendruckwerthen entsprechen. Als Material benützte Grünhagen Katzen und Kaninchen, die ex vor Beginn des Experiments mit Morphium narcotisirte. Er ging dann, als die Resultate zu unsicher wurden, zur Curarisirung der Thiere über. Die Canüle wurde 2,5 Millimeter in die Augenkammer vorgestossen. Veränderte sich nach dem Einstich der Stand des Quecksilbers, so konnte nicht sofort abgelesen werden, sondern es musste $\mathrm{Hg}$ so lange zu- oder abgegossen werden bis die Flüssigkeit zum Kammerwasser denselben Stand zeigte wie ror dem Einstich.

Wegner ging so ziemlich nach derselben Methode vor wie Grünhagen, nur fugte er zwischen Stichcanüle and Manometer einen Gummischlauch ein.

Adamük benutzte als Material Katzen und Hunde, fand als mittlere Druckhöhe bei Katzen 24,2 Millimeter Quecksilber. Das vordere Ende seines Manometers war in eine Spitze ausgezogen, welche dann durch eine vorgemachte Oeffinung in die vordere Augenkammer eingestossen wurde. Zur Narcose brauchte er Opium oder Chloroform. Adanük suchte ausser der Höhe des intraoculären Druckes den Einfluss von Nerven (Sympathicus) und Blutdruck auf die Augenspannung zu erforsehen.

Die manometrische Methode fand fernere Verwendung durch: 
Völckers und Hensen. *) Sie benutzten ein Wassermanometer und fanden als hauptsächlichstes Resultat, dass die Accommodation keine Aenderung der Druckhöhe wahrnehmbar mache.

von Hippel und Grünhagen veröffentlichten anno 1868 eine Arbeit: „Ueber den Einfluss der Nerven auf die Höhe des intraoculären Druckes." **) Diese benutzten nun statt Oel, wie früher Grünhagen (siehe oben), reines Wasser zwischen Quecksilber und Kammerflüssigkeit. Als Material wählten sie mit Vorliebe Katzen, weil deren Iris anf einen mechanischen Reiz nicht so prompt mit einer plastischen Entzündung reagire wie diejenige anderer Thiere. Resultate: Sympathicusreizung soll den Druck erhöhen. Der Ciliarmuskel soll gar keinen Einfluss haben auf die Höhe der Augenspannung.

Die Methode der manometrischen Messung wird beinahe gänzlich verworfen und nur so weit geltend gelassen, als man überhaupt Schwankungen des Drucks constatiren wolle von Stellwag von Carion. Er glaubt, sämmtliche physiologischen Verhältnisse im Auge seien mach Einstich der Canüle gestört und führt als Beweis für seine Behauptung gerade das Auftreten von Puls- und Athmungsschwankungen an. Er lässt sich über die verschiedenen Faktoren ans, welche die intraoculäre Spannung beeinflussen, und spricht mit v. Hippel und Grünhagen den intrabulbären Muskeln jede Druckbeeinfiussung ab. Ebenso spricht er sich verneinend über eine Alteration der Tension durch Myosis oder Mydriasis aus.

Das Mikro-Manometer von Hering benutzte Adamük za einer ferneren Reihe von Manometerversuchen. Diesem Instrument wird vorgeworfen, dass es in Folge von Capillar-Attraction kleine Drucksehwankungen nicht

*) "Studien über die Accommodation." Centralbl. für med. Wissensch. No. 46.

**) Archiv für Ophthalm. XIV. 5. 
zum Ausdruck zu bringen im Stande sei, und deshalb unrichtige Werthe gebe.

Leber hat sich viel mit manometrischen Messungen des Augendrucks abgegeben. Er bediente sich einer Canüle mit seitlich gelegener Oeffnung und war der Erste, der eine Contrapunction zum Experiment verlangte. Die Canüle wird horizontal durch eine seitliche Wand der vorderen Augenkammer durchgestossen, und die gegenüberliegende Cornealwand ebenfalls perforirt. Es trägt dies sehr zur guten Fixation der Canüle bei. Als wichtiges Erforderniss betrachtet Leber*) die rein conische Form der Canulle, damit das Auslaufen oder Absickern des Kammerwassers während des Einstichs verhindert werde.

Mit der Leber'schen Canüle hat auch E. Pflüger seine Manometerversuche an Kaninchen gemacht.

Eine wesentliche Verbesserung der Methode ist das Verdienst von Höltzke und Graser; beide haben zusammen über dasselbe Thema gearbeitet. Höltzke veröffentlichte ihre Resultate im Archiv für Ophthalmologie Bd. XXIX. unter dem Titel: „Experimentelle Untersuchungen über den Druck in der Augenkammer", und Graser denselhen Gegenstand im Archiv für experimentelle Pathologie und Pharmakologie Bd. XVII. Heft 5 unter dem Titel: „Manometrische Untersuchungen über den intraoculären Druck und dessen Beeinflussung durch Atropin und Eserin". Das Doppelmanometer, welches die Beiden in die Methode eingefuhrt haben, ist unbedingt ein schöner Schritt vorwärts. Damit ist dem Einwand, der bei allen frühern Manometerversuchen gemacht werden konnte, dass nümlich nach dem Einstich der Canüle von Flüssigkeit entweder etwas aus dem Auge heraus- oder in dasselbe hineingehen müsse, die Spitze gebrochen. Mit der sogenannten Compensationsschraube ist man

*) Archiv für Ophthalm. XIX. 2. 
sehr leicht im Stande, den dem Auge zugekehrten Schenkel, den innersten Quecksilberschenkel des Doppelmanometers, immer auf derselben Höhe zu haiten, auf der er vor dem Einstich der Canüle notirt ward. So wird es, wenn das ganze Röhrensystem im Uebrigen intact ist, unmöglich, dass etwas aus dem Bulbus in die Canüle oder umgekehrt übergehe, es ist also dadurch schon eine grosse Fehlerquelle der frühern Manometerexperimente beseitigt.

Eine fernere Vervollkommnung des Apparats haben Höltzke und Graser dadurch erwirkt, dass sie die Canüle mit einem Hartgummihalter versahen. In diesem ist ein Stopfstilet hin und her beweglich, welches, vorgestossen, vor dem Einstechen die Canüle vollkommen verschliesst, um nachher, wenn es zurückgezogen wird, vollkommene Communication zu schaffen.

Adamük und Monnik haben früher schon Messungen des Augendruckes im Glaskörper vorgenommen, diese Methode wurde aber bedeutend verbessert durch Schultén. Dieser maass den Druck manometrisch im Glaskörper und beobachtete zugleich das Verhalten der Netzhautgefüsse mit einem 30 bis $60 \mathrm{mal}$ vergrössernden Ophthalmoskope, welches auf einer combinirten Hohlspiegelaufstellung beruht. Die nähere Beschreibung des Instrumentes findet sich in Du Bois Reymonds Archiv für Physiologie 1882 Bd. XI. 3. und 4. Heft, sowie in der Schultén'schen Arbeit. Zu den Manometer-Experimenten brauchte er eine kurze, gebogene Stichcanüle von $3 / 4 \mathrm{Mm}$. Oeffnung; ein Gummischlauch setzt sie in Verbindung mit einem Manometer.

Zur nähern Orientirung über das Aussehen des ganzen Apparates verweisen wir auf die Arbeit von Schultén: „Experimentelle Untersuchungen über die Circulationsverhältnisse des Auges und über den Zusammenhang 
zwischen den Circulationsverhältnissen des Anges und des Gehirns." *)

Nachdem wir die Entwickelung der manometrischen Druckmessungsmethode kennen gelernt haben, wollen wir sehen, was für Ansichten über den Einfluss der Mydriotica und Myotica auf den intraoculären Druck bis jetzt aufgetaucht sind. Nicht Alle, jedoch die Meisten, die sich mit Messungen der Augenspannung abgegeben, haben es nicht unterlassen, das eine oder andere der cardinalen Heilmittel anf seine Beziehungen zum Binnendruck des Auges zu prüfen.

Adamück und Wegner (vergl. die oben angeführten Arbeiten) fanden, dass Atropin den Druck erniedrige; Ersterer überdies noch, dass Morphium und Opium sich dem Atropin gleich verhalten, Extractum fabae Calabaricae dagegen den intraoculären Druck steigere.

v. Hippel und Grünhagen sahen nach der Einträuflung von Atropin oder Aserin keine Veränderungen im Stand der Queeksilbersäule.

Ich stimme mit Graser vollkommen überein, wenn er hierin etwas Misstrauen Erweckendes puncto Versuchsanordnung sieht. Die Ausschläge des Quecksilbers sind denn doeh, wie wir später sehen werden, zu eclatant, wenn der Versuch sorgfältig and genau eingeleitet ist und glatt verläuft.

Stellwag von Carion spricht in seiner Arbeit Nichts von einem Einfluss des Atropin oder Eserin auf den intraoculären Druck.

Pflüger vergleicht in seiner Inauguraldissertation **) die Resultate der tonometrischen und manometrischen

*) Archiv für Ophthalm. Bd. XXX, Abtheil. 3, pag. 1-77 und Tafel I. Fig. 1 u. 2.

*) „Beiträge zur Ophthalmotonometrie“. Archiv f. Augenund Ohrenheilkunde II. 2. 
Ueber den Einfluss der Hydriatica und Myotica etc. 113

Methode bezüglich der Wirkung von Atropin auf die intraoculäre Spannung.

Einen völligen Umsturz der bis jetzt geltenden Ansichten über Atropin and Eserin und.deren Stellung zum Binnendruck des Auges vertrat Laqueur anno 1877 in einer Arbeit: "Ueber Atropin und Physostigmin und ihre Wirkung anf den intraoculären Druek."*) Er geht dabei von der Thatsache aus, dass Atropin das Glaucom im schlechten Sinne beeinflussen kann, und führt die Stimmen von Wharton Jones and A. v. Graefe an, von denen Ersterer vom Gebrauch des Atropins bei acutem Glaucom abräth, Letzterer latente chronische Glaucome durch Anwendung von Atropin in acute übergehen sah. Aehnliche Beobachtungen hätten H. Derby, Warlomont, Mooren, Hirschberg und Bezold gemacht.

Als Grund für diese drucksteigernde Eigenschaft des Atropins bei zu Glaucom disponirten Augen führt Laqueur einmal eine Hyperämie im Bereich des Ciliarkörpers und der Chorioidea an, weil die Iris im Zustand der Mydriase blutärmer werde als sonst. Für die Fälle, wo Drucksteigerung eintritt ohne Mydriase, wenn nämlich die Pupille durch circuläre Synechie fixirt ist, sieht er den Grund in einer Gefuss paralysirenden Wirkung des Atropins. Seine Ansicht ist im Schlusssatze des I. Theils der Arbeit enthalten: "dass das Atropin ein den intraoculären Druck erhöhendes Mittel ist, dass diese Wirkung aber nicht in die Erscheinung tritt, so lange die die Circulation regulirenden Einrichtungen gut functioniren.

Im II. Theil spricht Laqueur von den Versuchen, die er mit Eserin angestellt hatte. Er benutzte dazu das Dusquesnel'sche Physostigmin in Lösung von 0,01

*) v. Graefe's Archiv f. Ophthalm. Bd. XXIII. 3, p. 149.

v. Graefe's Archiv fïr Ophthalmologie, xxxim. 1. 
auf 2,5 Wasser. Ueber die Wirkung sagt er, dass dieses Alkaloid im normalen, menschlichen Auge den Druck nicht merklich herabsetze, auch im Kaninchenange sei nichts Erhebliches nachzuweisen. Laqueur sieht in der das Glaucom so gïnstig beeinflussenden Wirkung des Eserins im Gegensatz zum Atropin eine Contraction der Chorioidealgefässe und ,eine in Folge derselben verminderte Filtration in den Glaskörperraum". Aehnliches beobachtete man am Darm. Atropin lähmt den Darm einer Katze, eine gleich darauf gemachte Physostigmin-Injection dagegen erzeugt eine lebhafte Peristaltik.

Auf dem Ophthalmologen-Congress in Mailand, 1880, hielt Pfluger einen Vortrag: Des oscillations artificielles de la tension intraoculaire à l'état physiologique."*) Diesem entnehmen wir Folgendes.

Pflüger referirt hier aber seine Kaninchenversuche, bei denen er den Anweisungen, welche Leber hierüber gegeben, gefolgt sei. Er untersuchte zuerst am nicht curarisirten, dann am curarisirten Thier, führte an jedem Auge eine Leber'sche Canüle durch die vordere Augenkammer und stellte ein Auge unter die Einwirkung des Medikaments, das andere nicht. Katzen hielt er nicht für nöthig. Seine Resultate sind: Mittlerer Druck im Kaninchenauge $18 \mathrm{Mm}$. Quecksilber. Differenzen in beiden Manometern verschwinden nach 10 bis 20 Minuten. Atropin giebt 10 bis 15 Minuten nach der Einfuhrung der Canüle 4 , höchstens 5 Millimeter Druckherabsetzung in einem Fall gar keine Herabsetzung, jedoch niemals Erhöhung. Weil Eserin die Tritis plastica durch Zusammenziehung der Pupille begünstigt, sind die Veqruche mit diesem Medikament nicht so gleichförmig. Nach 15 bis 45 Minuten sah Pflüger 2-4 Mm. Erhöhnng, ein Mal

*) Congrès periodique international d'ophthalmologie, VI. Session. Compte-Rondu par Mons. le Dr. Pierdhouy, secrét, 1880. 
um $9 \mathrm{Mm}$; in zwei Fällen' gar kein Einfluss. Bis jetzt hat er keine constante, evidente Herabsetzung des Augendrucks durch Eserin beobachtet.

Sehr in Zweifel gesetzt wurde die Wirkung des Atropins als Druckverminderer durch A. Weber.*) Er behauptet, Atropin setze im Glaskörper den Druck herab, im vordern Kammerraum dagegen erhöhe es denselben stets. Vom Eserin will er immer bedeutende Druckerniedrigung in der vorderen Kammer gesehen haben.

Im Laufe des Jahres 1882 erschien eine Dissertation von W. Reinhardt, betitelt: „Vorderer Kammerdruek und Substanzverluste der Cornea unter Atropin and Eserin". Der Verfasser gelangt an Hand anderer Experimente zu den gleichen Resultaten wie Weber und sagt, Atropin vermehre den Druck in der vorderm Kammer, Eserin vermindere denselben. Fr erzeugte an Kaninchenaugen durch Abkratzen der äussersten Schichten der Cornea Geschwüre und beobachtete deren Verhalten unter Einträuflung von Atropin und Eserin. Anf dem Eserin-Auge sah er nach 24 Stunden den Geschwürsgrund weniger vorgebuchtet, das Geschwür hatte sich verkleinert, und beim Abkratzen des Geschwürgrundes mit der Lanze bemerkte er eine geringere Resistenz als auf dem Atropinauge. Diese Unterschiede waren nach 24 Stunden ausgesprochener als nur kurze Zeit nach der Instillation, obschon Myosis und Mydriasis beinahe verschwunden war. Träufelte er nach dieser Zeit wiederum Eserin und Atropin ein, so sah er, dass, wann Myose und Mydriase ihr Maximum erreicht hatten, die Resistenz des Geschwürgrundes auf der Eserinseite grösser geworden war als auf der Atropinseite, obschon or sich mebr abgeflacht hatte.

*) "Ueber Calabar und seine therapeutische Verwendung." Archiv f. Ophthalm. Bd. XXIII. 
Anknüpfend an diese letztere Beobachtung Reinharăts wies Pflüger auf der vierzehnten Versammlung der ophthalmologischen Gesellschaft in Heidelberg, 1882*), in seinem Vortrag: "Zur Behandlung des Glaucoms" nach, dass die Kriterien, welche Reinhardt für die veränderte Tension aufführt, in Wirklichkeit wenig mit derselben $\mathrm{zu}$ schaffen haben, sondern zum guten Theil wenigstens auf Quellungsverhältnisse der anliegenden und neu sich bildenden Hornhautsubstanz zu beziehen sind. Gleichen Orts weist Pfinger, gestutzt auf seine Manometerversuche, die Behauptungen Webers zurück. Ueber seine neuesten Versuche mit Eserin erzählt er, dass nach 15-20 Minuten stets eine Steigerung des Drucks ungefähr um 4-6 Millimeter eingetreten sei. Der Versuch konnte, wie fruher, nicht äber eine Stunde ausgedehnt werden wegen der die Canüle mit Exsudat verstopfenden Iritis plastica. Zum ersten Male brachte hier Pflüger seine Beobachtungen über Pilocarpin vor; 5 Minuten nach der Einträufelung ins Auge eines nicht curarisirten Thieres sah er während grosser Unruhe des Thieres rasche Schwankungen der Quecksilbersïule. Nach 25 Minuten begann die Tension zu sinken von einem Anfangsdruck von 22 Otecksilbermillimeter auf 20 , dann anf $19 \mathrm{Hg}$. Mm., nach 50 Minuten auf $18 \mathrm{Hg} . \mathrm{Mm}$. Und so proclamirt er das Pilocarpin als ein den intraoculären Druck primär herabsetzendes Mittel. Im Resumé seines Vortrages kommt Pflüger zum Schluss **), „dass die Wirkung der Mydriatica und Myotica eine andere unter physiologischen und eine andere unter gewissen pathologischen Bedingungen sein muss, dass in gewissen glaucomatösen Zuständen Effecte dieser Substanzen zur Geltung kommen, welche normaliter den intraoculären Druck nicht beeinflussen."

*) Bericht über dieselbe, redigirt durch $F$. C. Donders, W. Hess and W. Zehnder. pag. 137.

*to) Obiger Bericht pag. 142. 
In der anschliessenden Discussion der Versammlung vertheidigt Laqueur seine oben angeführten Thesen und gibt eine Kritik der Thierexperimente, indem er im Einführen einer Canüle eine Reizung und Störung der laumverhältnisse der vordern Augenkammer sieht. Ferner begreift er nicht die verschiedene Wirkung von Eserin und Pilocarpin.

So war der Stand der Frage betreffend den Einfluss von Atropin und Eserin auf die Augenspannung: Pflüger hatte, geleitet von den Resultaten seiner Manometerversuche, von seinem Satze nie abgelassen, dass Atropin den Druck im physiologischen Auge erniedrige, während Laqueur, gestützt auf die guten Wirkungen des Eserins bei Glaucom und die oft schlimmen Erfolge mit Atropin bei derselben Krankheit, behauptete, Eserin setzt den Druck im Auge herab, Atropin erhöht ihn.

Jetzt traten Höltzke und Graser mit ihrer epochemachenden Arbeit an die Oeffentlichkeit. Ihre Verdienste um Verbesserung der manometrischen Druckmessungsmethode haben wir bereits oben erwähnt; die Schlüsse, welche sie aus ihren Manometerversuchen zogen, waren folgende:

Höltzke sagt im Arch. f. Ophthalmologie Bd. XXIX. Abth. II. pag. 11:

1. „Mit der durch Atropin bewirkten Erweiterung der Pupille geht Hand in Hand eine Steigerung des Drucks in der Kammer, und mit der durch Eserin bewirkten Verengerung eine Herabsetzung desselben."

2. "Eserin erhöht den Druck in der Kammer ete."

Graser in seinem ausführichen Bericht im Archiv für experimentelle Pathologie und Pharmakologie Bd. XVII. H. 5. pag. 357 u. folg.:

1. „Die manometrische Untersuchungsmethode ist für 
die Bestimmung des intraoculären Drucks, besonders aber für Bestimmungen von geringen Schwankungen nicht nur ausreichend, sondern besonders in der zuletzt berührten Hinsicht wohl das einzige Mittel, um genaue Resultate zu erhalten."

2. ,Im wesentlichen ist die Höhe des intraoculären Drucks abhängig von dem jeweiligen Blutdruck."

3. „Die absolute Höhe des intraoculären Drucks steigt ziemlich proportional mit der Grösse des Versuchsthiers derselben Species ...... als Durchschnittshöhe des Drucks gewannen wir aus 35 Beobachtungen für mittelgrosse Katzen etwa 28 Hg-Mm."

4. „Das Spiel der Irismuskulatur äussert auf die Höhe des intraoculären Druckes einen bestimmten Einfluss in der Art, dass die Erweiterung der Pupille eine Steigerung, Verengerung eine Herabsetzung der Druckhöhe bedingt."

5. „Atropin, in der gewöhnlich zur Herbeifübrung von Mydriasis gebrauchten Dosis in den Bindehautsack gebracht, erhöht den intraoculären Druck."

6. "Eserin, in gleicher Weise angewendet, bedingt zunächst eine Drucksteigerung; der aber in allen Fällen nach Verlauf von spätestens einer Stunde (nachdem sich Myosis entwickelt) eine Drnckerniedrigung unter die Norm nachfolgt."

Diese Resultate der Höltzke-Graser'schen Versuche sind meines Wissens nicht mehr geprüft worden, sondern sie wurden als unantastbar so ziemlich von der ophthalmologischen Welt acceptirt und der Satz: "Atropin erhöht, Eserin erniedrigt den intraoculären Druck", ging schon in physiologische Lehrbücher*) über.

*) Vergl. Landois. 
Mein hochverehrter Lehrer, Herr Professor Dr. Pflüger, der bei seinen zahlreichen, frühern Manometerversuchen am Kaninchen gerade das Gegentheil stets wahrgenommen hatte, konnte sich begreiflicher Weise nicht bei der blossen Annahme dieser Sätze beruhigen, er wollte die Sache nochmals von dritter Hand geprüft wissen. Es schien ihm die Frage, ob das mydriatische Princip der Solaneen unter physiologischen Verhältnissen den intraoculären Druck in positivem oder negativem Sinne beeinflusse, für das therapeutisehe Handeln des Ophthalmologen, der sich keinen Tag der Mydriatica entschlagen kann, zu wichtig, als dass man einen Zweifel in derselben unaufgeklärt bestehen lassen dürfte. So verdanke ich ihm denn die Anregung zur vorliegenden Arbeit.

Im letzten Herost machte ich zur Einübung der Technik viele Versuche an Kaninchen, welche mit der von Holtzke und Graser benutzten Billrothschen Mischung *) narkotisirt wurden. Ich hielt mich bei diesen Experimenten ganz an die schon längst bekannten Vorschriften, und die namentlich auch von Graser betont wurden: Es soll keine Luftblase sich im ganzen System der Röhren befinden, kein Tropfen Kammerwasser soll abfliessen, die Iris beim Einstich nicht gestreift werden. Als mittlerer Druck bei Kaninchen fand ich 18-20 Mm. Hg.

Was die Streitfrage anbetrifft, ob man in der vordern Augenkammer messen dürfe, um daraus einen Sehluss über den gesammten Binnendruck des Auges zu zishen, da ja der Druck im Glaskörper verschieden sein könne, so glaube ich, die Sache sei seit Schultén ${ }^{* *}$ ) so ziemlich abgethan. Dieser hat nämlich vergleichende Messungen angestellt im Glaskörper und der vordern Kammer und sehr kleine Differenzen beider Druckgrössen gefunden, und

*) Chloroform 200,0, Alcohol. absol., Aeth. sulph. âa 50,0.

**) Archir für Ophthalm. Bd. XXX. 3. pag. 34. 
sagt: „Druckveränderungen durch Flüssigkeits-Injection in die vordere Kammer oder den Glaskörper bewirken eine parallele Steigerung in beiden Augenabtheilungen".

Mit Beginn des letzten Wintersemesters wurde meine Arbeit in hohem Maass dadurch gefördert, dass mir von Herrn Professor Dr. Kronecker in der zuvorkommendsten Weise ein Zimmer auf dem physiologischen Institute eingeräumt wurde. Als Material benutzte ich von nun an Katzen, die übrigens, heiläufig bemerkt, in Bern ein äusserst rarer Artikel sind, wohl wegen des in allen Klassen der Bevolkerung Fuss fassenden AntivivisectionsFanatismus.

Zuerst versuchte ich zur Immobilisirung die Chloroform-Morphiumnarkose, wie sie Graser und Höltzke besorgt hatten. Obschon ich aber ganz reine Präparate von Morphium benutzte, obschon die Billroth'sche Mischung frisch aus der Bernischen Staatsapotheke bereitet war, gehörte es zu einem Ding der Unmöglichkeit, irgend eine der Katzen nur für eine Viertelstunde in eine so ruhige Narkose zu bringen, wie sie unsere Experimente verlangen; von einer Einschläferung mit 0,04 Gr. Morphium, subcutan injieirt, wie sie Graser glatt bekommen hat (vergl. die Arbeit pag. 348), war nun schon gar keine Rede. Ein Kater hat 0,07 Gr. Morph. subeutan und darauf eine volle halbe Stunde Billroth'sche Mischung ertragen, ohne zu schlafen; es war vollkommen das Bild der Narkose eines Potatoren. Nach wiederholten vergeblichen Versuchen stand ich von der Narkotisirung mit Morph. und Chloroform $a b$ und ging zum Curare über. Von da an verlief mir jeder Versuch, was die Narkose anbelangte, ganz glatt mit Ausnahme eines einzigen, in welchem das Thier starb, weil wir wahrscheinlich unreines (strychninhaltiges) Curare benutzt hatten.

An dem Canülenhalter aus Hartgummi, welcher sammt den Canülen genau nach der Graser'schen Vorschrift von 
Ueber den Einfluss der Mydriatica und Myotica etc. 121

Reiniger in Erlangen bezogen wurde, brachte Pflüger eine kleine Verbesserung an, die uns sehr zu Statten kam. Es ist Graser und auch mir, trotz aller Vorsicht bei der Füllung, Ansangung der Canüle und Befestigung des

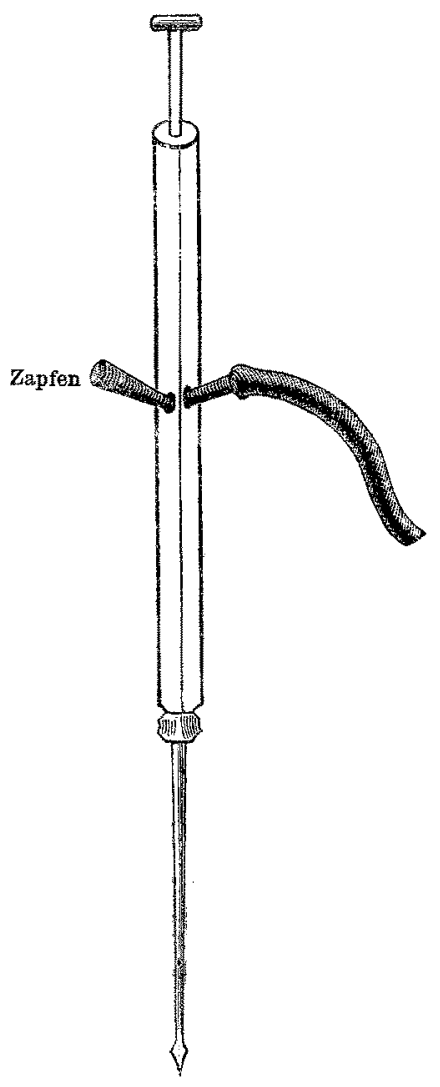

Gummischlauchs am Manometer, öfter vorgekommen, dass sich dennoch eine Luftblase ins Röhrensystem verirrte. Dies ist bei Benutzung der Pfiüger'schen Modification des Hartgummihalters nicht mehr möglich. Auf der Seite, die derjenigen anliegt, welche den Ansatz für den Gummi- 
schlauch trägt, ist eine Oeffnung gebohrt, welche mit dem Hohlraum des Canulenhalters communicirt und mit einem Gewinde versehen ist, in welches die Schraube des luftdicht versehliessenden Zapfens passt.

Wenn nun alles gefüllt und zum Einstich bereit ist, wird, bevor man den künstlichen Ueberdruck im Nanometer erzeugt, der Zapfen, nachdem der Canülenhalter möglichst hoch gehalten wird, losgeschraubt und durch leises Beklopfen des Letzteren auch das letzte Luftbläschen zur Oeffnung herausgetrieben. Darauf wird der Zapten wiederum eingeschraubt und man ist sicher, im Versuche nicht durch das sehr ärgernde Auftauchen einer Luftblase gestört zu werden.

Die ursprünglichen Canülen, welche vom Universitätsmechaniker Reiniger in Erlangen geliefert wurden, waren zu eng, ich möchte fast sagen zu capillar ausgefallen, da sie bei hohem Ueberdruck im Manometer und bei zurückgezogenem Stilet die Flüssigkeit nicht, wie es sein soll, in feinem Strahl, sondern träge, tropfenweise austreten liessen.

Ich liess zwei neue Canülen anfertigen bei der Firma Hauhart \& Klopfer in Bern, an denen auch die seitliche Oeffnung so angebracht wurde, dass - damit sie sich vis-à-vis der Pupillenmitte befinde - bei der Contrapunction die ganze stählerne Spitze aus der Cornea herausragen musste.

So hatte man einen Anhaltspunkt, dass die Canüle und damit ihre Oeffnung genügend in der Kammer vorgeschoben war.

Die Fehlerquelle des elastischen Schlauches suchten wir zu vermeiden, indem wir entweder in einen etwas weitern Gummischlauch Glasröhrenstückchen von $2-3 \mathrm{Cm}$. Länge und entsprechender Dicke einschoben und so den grössten Theil des nachgiebigen Gummirohres ausschalteten; oder wir benutzten einen ganz starren Schlauch von 
Ueber den Einfluss der Mydriatica nnd Myotica etc. 123

entsprechendem Kaliber. Auf diese Weise vermieden wir, dass die Flüssigkeit in das elastische Rohr gepresst wurde.

Dies erkennt man daran, dass der dem Thierauge zugekehrte Quecksilberschenkel - man mag den Druck mit der Compensationsschraube erhöhen so viel man will nicht seine Stellung wechelt, wenn das Stilet der Canüle vorgestossen ist.

Als Füllungsflüssigkeit des Manometers, des Schlauchs und der Canüle benutzten wir die physiologische Kochsalzlösung $(0,6 \%)$. Zur genaueren Ablesung der Scalatheile der Manometer wurde ein Fernrohr mit Fadenkreuz vis-à-vis aufgestellt. Es ist dies, namentlich wo es sich um kleine Schwankungen der Quecksilbersäule handelt, sehr zu empfehlen, da es der Parallaxe wegen von blossem Auge schwierig ist, die Stellung der Quecksillberkuppe zu den Scalatheilen richtig und genau herauszubekommen. Die Ablesungen wurden, wenn nichts Besonderes bemerkbar war, von fünf zu fünf Minuten besorgt, die Quecksilbersiulule jedoch stets im Auge behalten.

Der Einstich wurde so schnell als möglich auf einem Auge nach dem andern gemacht. Gleich nacher zeigten sich immer bedeutende Schwankungen der Druckhohe beider Augen, so dass man vollauf zu thun hatte, den innersten (d. h. den dem Auge des Versuchsthieres zugekehrten) Quecksilberstand immer auf derselben Höhe zu halten. Diese Schwankungen, wahrscheinlich eine Folge des mechanischen Reizes von Seite der durch die Kammer gedrungenen Canüle hören je nachdem bald nach einer Viertel- oder halben Stunde auf. Dann zeigt sich unser physiologischer Augendruck. Wir haben es of gesehen, dass dieser beim Nillimeter gleich war auf beiden Angen während 10 Minuten oder einer Viertelstunde, oft war ein bis zwei Millimeter Untersebied. Man kann auch nicht verlangen, dass die intraoculäre Spannung beiderseits 
haarscharf dieselbe sei, ist doch der Blutdruck in den Carotiden nicht einmal physiologischer Weise beidseitig genau gleich. Man könnte nun wiederum gegen die Methode der Messung mittelst Durchstechen einer Hohlnadel einwenden, dass gerade die starken Schwankungen des Hg nach dem Eingriff beweisen, wie hier das physiologische Gleichgewicht gestört ei. Das alles zugegeben, verbietet uns nichts, richtige Schlüsse zu ziehen aus dem Verhalten der Quecksilbermanometer anf den Einfluss eines Arzneimittels auf den Augendruck, wenn das Auge, das dem einen Manometer zugehört, nur von der Canüle, das andere aber von der Canüle plus dem Medikament alterirt wird. Wir wollen ja dabei nicht absolute, sondern nur ganz relative Werthe. Das ist jedoch unbedingt nothwendig, dass beide Augen - und zwar gleichzeitig der Beobachtung unterworfen werden, damit das eine uns immer den sogenannten physiologischen Druck präsentire. Ohne diese Vorsichtsmaassregeln ist jede Beobachtung werthlos, denn wir werden an einzelnen Versuchen sehen, wie variabel, ja hüpfend die Curve des physiologischen Druckes sein kann, und wird ein Auge allein beobachtet, ist es nicht anders möglich als solche Druckunterschiede auf Rechnung einer Medikamentwirkung zu registriren. So haben wir denn aus keinem Versuche irgend welche Schlussfolgerung, betreffend die Wirkung eines Mydriaticum oder Myoticum, gezogen, bei dem nicht das eine Auge das controlirende, physiologische war.

Ich kann um so eher Umgang nehmen von der schematischen Darstellung eines Versuchs, als mit Ausnahme der bereits erörterten Modificationen die Anordnung des Experiments nach dem Typus Höltzke-Graser*) war.

*) Archiv f. experiment. Path. und Pharmakolog. Bd. XVII. H. V, p. 346 . 
Was die Pols- und Athmungssehwankungen der Manometerquecksilbersäule betrifft, möchte ich behaupten, dass diese für uns beim Experimente etwas Unentbehrliches sind, obschon Stellwag von Carion, der uberhaupt die manometrische Methode in ihrem Werthe unterschätzte, glaubte, diese Schwankungen bedeuten gestörte physiologische Verhältnisse im durchstochenen Auge. Diese durch Respiration und Herzschlag hervorgebrachten Schwankungen der Manometersäule waren für uns immer das beste Zeichen, dass mit einem Worte, Alles in Ordnung, die Communication der Flüssigkeiten vollkommen frei, ungestört von Luftblasen, Exsudat etc. war. Ferner haben wir bemerkt, dass die Puls- und Athmungsschwankungen nur bei einer gewissen normalen Tension des Auges, bei kräftiger Herzaction vorkamen; ihr Verschwinden oder Undeutlichwerden war ein erstes Symptom des herannahenden Exitus. Die Athmungsschwankungen gaben Excursionen des Quecksilbers von einem halben bis einem Millimeter, die Pulssehwankungen von der Distanz, von Hg-Meniscus bis Hg-Kuppenhöhe. Die Druckhöhe im (curarisirten) Katzenauge fanden wir zwischen 25 und 32 Quecksilbermillimetern, bei kräftigen Thieren höher, bei sebwächern geringer.

Indem wir nun zu den Versuchen mit den einzeInen Arzneimitteln im Speciellen übergehen, werden wir jedes Alcaloid gesondert betrachten an Hand der einschlägigen Versuchsprotokolle.

\section{A tropin.}

Wir benutzten zur Einträufelung in den Bindehantsack eine 1 procentige Lösung von Atropinum sulphuricum. Die Instillation musste bei allen Arzneimitteln öfters wiederholt werden, weil der grösste Theil der Flüssigkeit 
abtropfte, namentlich wenn man mach dem Vorgang von Höltzke-Graser die äussere Commissur schlitzte, was allerdings Fixation des Bulbus und Einstich der Canüle erleichtert. Die Bulbusoberfläche sowohl als die Stichcanüle wurden stets mit 5 procentiger Resorcinlösung desinficirt.

Wir wollen zuerst einen der besten Typen der Atropinversuche aufführen, welcher der grossen Mehrzahl der bezugglichen Experimente entspricht. Es ist dies der Versuch vom 23. März 1885.

Ausgewachsene Katze. 0,01 Curare in die Vena jugularis externa, vorher 0,01. Morph. subcutan.

Wir notiren hier gleich den ausgerechneten Worth für dio betreffende Druckhöhe, indem wir den notirten Stand des innersten Schenkels weglassen.

\begin{tabular}{c|c}
$\begin{array}{c}\text { Rechtes Auge. } \\
\text { Zordruck }): 32 \mathrm{Mm} .\end{array}$ & $\begin{array}{c}\text { Linkes Auge. } \\
\text { Vordruck: 32 Mm. } \\
\text { Zeit } \mid\end{array}$ \\
\hline & $\mid$
\end{tabular}

Nach Herstellung der Communication:

Pulsw und Athmungsschwankungen sehr dentlich.

\begin{tabular}{|c|c|c|c|c|c|c|}
\hline \multicolumn{3}{|c|}{$34 \mathrm{Hgmm}$. } & \multirow{3}{*}{$10 \mathrm{~h} 40 \mathrm{~m}$} & \multirow{2}{*}{\multicolumn{3}{|c|}{$28 \mathrm{Hgmu}$. }} \\
\hline $10 \mathrm{~h} 40 \mathrm{~m}$ & 34 & $"$ & & & & \\
\hline $10 \mathrm{~h} 50 \mathrm{~m}$ & 32 & $*$ & & Pup.mittelw. & 29 & $\%$ \\
\hline $10 \mathrm{~h} 55 \mathrm{~m}$ & Pup. mittelw, 32 & $"$ & $10 \mathrm{n} 50 \mathrm{~m}$ & & 32 & $n$ \\
\hline $11 \mathrm{~h}-\mathrm{m}$ & 30 & $"$ & $10 \mathrm{~h} 55 \mathrm{~m}$ & & 30 & $"$ \\
\hline $11 \mathrm{~h} 5 \mathrm{~m}$ & 30 & " & $11 h-m$ & & 30 & " \\
\hline $1 \mathrm{~h} 10 \mathrm{~m}$ & 30 & $"$ & $11 \mathrm{~h} 5 \mathrm{~m}$ & & 30 & " \\
\hline $11 \mathrm{~h} 15 \mathrm{~m}$ & 30 & $"$ & $11 \mathrm{~h} 10 \mathrm{~m}$ & A tropin & 30 & 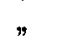 \\
\hline $11 \mathrm{~h} 20 \mathrm{~m}$ & 30 & $"$ & $11 \mathrm{~h} 15 \mathrm{~m}$ & & 27 & $\Rightarrow$ \\
\hline
\end{tabular}

*) Der Vordruck wird gemacht indem, wenn bei geschlossener Canüle der Hg-Stand im 1. und 4. Schenkel des Doppelmanometers notirt ist, durch Drehung der Compensationsschraube das Quecksilber um die Hälfte der gewünschten Millimeter über den notirten Stand des 4. Schenkels hinaufgetrieben wird. 
Teber den Einfluss der Mydriatica und Nyotica etc. 127

\begin{tabular}{|c|c|c|c|c|c|}
\hline \multicolumn{2}{|c|}{$\begin{array}{l}\text { Reehtes Auge. } \\
\text { Vordruck: } 32 \mathrm{Mm} \text {. }\end{array}$} & \multicolumn{4}{|c|}{$\begin{array}{l}\text { Linkes Auge. } \\
\text { Vordruck: } 32 \mathrm{Mm} \text {. }\end{array}$} \\
\hline $11 \mathrm{~h} 25 \mathrm{~m}$ & $\begin{array}{l}30 \mathrm{Hgmm} \text {. } \\
\text { Thier zuck }\end{array}$ & $\begin{array}{l}11 \mathrm{~h} 20 \mathrm{~m} \\
11 \mathrm{~h} 25 \mathrm{~m} \\
\text { t. Curare }\end{array}$ & & $\begin{array}{l}26 \mathrm{H} \\
26\end{array}$ & $\mathrm{gmm}$ \\
\hline $11 \mathrm{~h} 30 \mathrm{~m}$ & $32 \mathrm{Hgmm}$. & $11 \mathrm{~h} 30 \mathrm{~m}$ & Atropin & 28 & $"$ \\
\hline $11 \mathrm{~h} 35 \mathrm{~m}$ & $29 \#$ & $11 \mathrm{~h} 35 \mathrm{~m}$ & Pup. $8 \mathrm{Mm}$. & 30 & $n$ \\
\hline $11 \mathrm{~h} 40 \mathrm{~m}$ & 32 & $11 \mathrm{~h} 40 \mathrm{~m}$ & & 30 & " \\
\hline $11 \mathrm{~h} 45 \mathrm{~m}$ & 32 & $11 \mathrm{~h} 45 \mathrm{~m}$ & & 30 & $"$ \\
\hline $11 \mathrm{~h} 50 \mathrm{~m}$ & 32 & $11 \mathrm{~h} 50 \mathrm{~m}$ & & 29 & ” \\
\hline $12 \mathrm{~h}-\mathrm{m}$ & 32 & $11 \mathrm{~h} 55 \mathrm{~m}$ & & 28 & $"$ \\
\hline $1.2 \mathrm{~h} 5 \mathrm{~m}$ & 32 & $12 \mathrm{~h}-\mathrm{m}$ & & 27 & $"$ \\
\hline $12 \mathrm{~h} 8 \mathrm{~m}$ & 82 & $12 \mathrm{~h} 5 \mathrm{~m}$ & & 27 & $"$ \\
\hline $12 \mathrm{~h} 18 \mathrm{~m}$ & 32 & $12 \mathrm{~h} 10 \mathrm{~m}$ & & 26 & $"$ \\
\hline $12 \mathrm{~h} 24 \mathrm{~m}$ & $32 n$ & $12 \mathrm{~h} 15 \mathrm{~m}$ & $\begin{array}{l}\text { Atropin } \\
\text { Pup. } 9 \mathrm{M}\end{array}$ & 26 & $"$ \\
\hline $\begin{array}{r}12 \mathrm{~h} 30 \mathrm{~m} \\
\text { Pupille }\end{array}$ & immer gleich weit. & $12 \mathrm{~h} 20 \mathrm{~m}$ & & 26 & $n$ \\
\hline $12 \mathrm{~h} 40 \mathrm{~m}$ & $32 \mathrm{Hgmm}$ & $12 \mathrm{~h} 25 \mathrm{~m}$ & & 26 & " \\
\hline $12 \mathrm{~h} 45 \mathrm{~m}$ & $32 \cdot \pi$ & $12 \mathrm{~h} 30 \mathrm{~m}$ & & 24 & $"$ \\
\hline $12 \mathrm{~h} 47 \mathrm{~m}$ & 32 & $12 \mathrm{n} 35 \mathrm{~m}$ & Pupillo ad $x$ & 24 & $" m$ \\
\hline $\begin{array}{l}12 \mathrm{~h} 50 \mathrm{~m} \\
\text { Puls- und } \\
\text { gen fange }\end{array}$ & $\begin{array}{l}\text { A2 } \\
\text { Athmungsschwankun- } \\
\text { an undeutlich zu } \\
\text { werden. }\end{array}$ & $\begin{array}{l}12 \mathrm{~h} 40 \mathrm{~m} \\
12 \mathrm{~h} 45 \mathrm{~m} \\
12 \mathrm{~h} 50 \mathrm{~m} \\
12 \mathrm{~h} 55 \mathrm{~m}\end{array}$ & erweitert & $\begin{array}{l}23 \mathrm{H} \\
23 \\
23 \\
22\end{array}$ & gmin. \\
\hline & uls langsam, klein, Th & er.der Ver & giftung nal & & \\
\hline $\begin{array}{l}1 \mathrm{~h}-\mathrm{m} \\
1 \mathrm{~h} 20 \mathrm{~m}\end{array}$ & $\begin{array}{l}26 \text { Hgmm. } \\
26 \quad "\end{array}$ & $\begin{array}{c}1 \mathrm{~h}-\mathrm{m} \\
\text { Das Queo } \\
\text { tem }\end{array}$ & $\begin{array}{l}\text { ksilber ist } \\
\text { Sinken beg }\end{array}$ & $\begin{array}{l}21 \\
\text { in con } \\
\text { riffen. }\end{array}$ & istan. \\
\hline
\end{tabular}

Hier wurde der Versuch abgebrochen.

Bei Angabe der Pupillenweite ist entweder durch KD der kleinste Durchmesser and GD der grösste bezeichnet, da bekanntlich bei den Katzen die Myose und Mydriase vom Centrum zur Peripherie und umgekehrt nicht gleichmässig vor sich geht. Ist keine nähere Angabe gemacht, so ist immer der kleinste Durchmesser gemeint. Wie in diesem Versuche, so haben wir immer erst dann das Medikament auf das betreffende Auge einwirken lassen, wenn 
auf beiden Angen der Druck etwa zehn Minuten bis eine Viertelstunde gleich hoch geblieben war. Es war dies ein Zeichen, dass die dureh Einführung der Canüle verursachten Druckschwankungen sich verloren hatten. Es fand die erste Atropin-Einträufelung in das linke Auge statt, nachdem auf diesem von $10 \mathrm{~h} 55 \mathrm{~m}$ bis $11 \mathrm{~h} 10 \mathrm{~m}$ der Druck immer $30 \mathrm{Hg}$-Mm. betragen und aut dem rechten

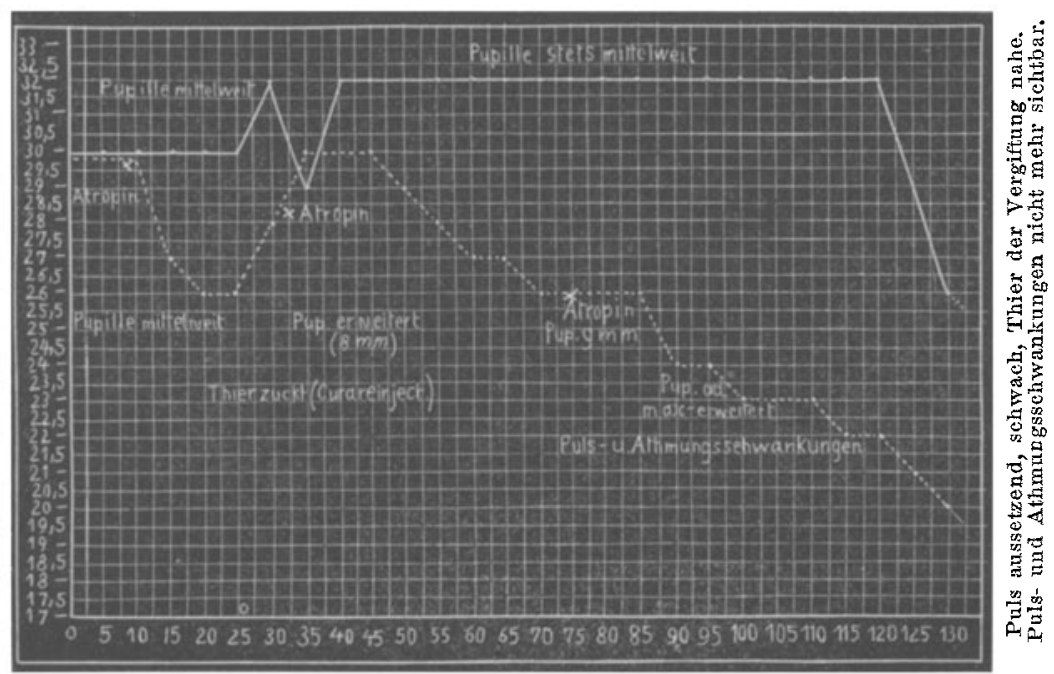

Figur 1.

Auge die Tension von $11 \mathrm{~h}$ bis $11 \mathrm{~h} 10 \mathrm{~m} 30 \mathrm{Hg}$-Mm. notirt hatte.

Ich habe diese Experimente durch Curven anschaulicher zu machen versucht, und zwar auf vorstehender Zeichnung (Fig. 1).

Die weiss gezogenen Linien entsprechen der sogen. physiologischen Curve auf dem durch kein Medicament beeinflussten rechten Ange, die punktirten der Atropincurve des linken Auges. Auf der Abscisse ist in sämmtlichen Tafeln die Beobachtungszeit von fünf $z \mathfrak{u}$ fünf 
Minuten angemerlit, der Zeit von fün Minuten entspricht das Spatium von zwei Theilungen des carrirten Papieres. Auf der Ordinate entspricht je ein Papiertheilstrich einem halben Hg-Mm.-Druck im Manometer, die Curvenlinien sind nach den im Versuchsprotokoll notirten Zeiten und Druckhöhen eingezeichnet.

Man sieht, dass während zehn Minuten beide Drucke gleich hoch sind, dann geht derjenige der Atropinseite nach der Eintrăufelung auf $26 \mathrm{Mm}$. hinunter, um aber nach 25 Minuten wieder bis zu $28 \mathrm{Mm}$. zu steigen. Auf Erneuerung der Instillation steigt die Curve bis $30 \mathrm{Mm}$; diese letzte Steigung von $26 \mathrm{Mm}$. bis $30 \mathrm{Mm}$. fallt mit einer Steigerung des physiologischen Druckes zusammen, der bis dahin immer auf $30 \mathrm{Mm}$. gestanden hatte. Als Ursache dieser Steigerung ist Zuckung des Thieres notirt, da nämlich die Curarewirkung schwächer geworden. Solche Zuckungen zeigen sich im Masometer immer als Drucksteigerung. Die darauf folgende Curare-Injection erniedrigt den physiologischen Druck momentan, was wir stets beobachtet haben. Während sich nun die Pupille des linken Auges zu erweitern beginnt, ist der Druck der physiologischen Seite wieder auf $32 \mathrm{Hg}-\mathrm{Mm}$. gestiegen, wo er nun als schöne Controle während des ganzen Versuches verharrt. Fünfundzwanzig Minuten nach der ersten Atropin-Einträufelung sinkt der Druck des betreffenden Auges von 1 bis $3 \mathrm{Mm}$. terassenförmig ab, bleibt auf $26 \mathrm{Mm}$. während einer Viertelstunde stehen, um nach nochmaliger Atropingabe bis zu $22 \mathrm{Mm}$. hinunter zu steigen. Hier finden wir ihn zu der Zeit, wo das plotzliche Abfallen der physiologischen Curve uns den nahen Tod des Thieres ankündigt; da sinkt auch auf der linken Seite das Quecksilber immer mehr. Wir sehen also aus den Curven, dass Atropin auf dem linken Ange eine langsame Herabsetzung des Augendruckes bewirkt hat. Das Maximum der Depression (10 Hg-Mm.) 
erreicht die Curve 1 Stunde und 50 Minuten nach der ersten Atropin-Instillation.

Obschon dieser Versuoh in jeder Beziehung tadellos verlaufen war, würde er allein nichts bedeuten, es könnte ja einmal aus irgend einem unbekannten Grunde der Druck sonst gefallen sein, während er auf dem andern Auge hoch geblieben. Jedoch war es überhaupt interessant für uns, bei jedem Manometerversuch mit Atropin die Druckerniedrigung eintreten zu sehen, namentlich da sonst überall zu lesen war, Atropin erhôhe den Druck. Neben den maassgebenden Versuchen mit Controle von Seite des andern nicht vergifteten Auges, haben wir verschiedene Beobachtungen an einem Auge gemacht, wenn das andere aus irgend einer Ursache bei der Einleitung des Experiments verunglückte. - Jedesmal ist der Druck gesunken. Diese Beobachtungen, so bedeutungslos sie alleinstehend wären, werden selır beweisend und unterstützend durch die Doppelversuche auf zwei Augen.

Von einem der letzteren Art soll noch das Protokoll mitgetheilt werden; die dazu gehorige Curve weist Fig. 2 auf.

XV. Versuch vom 25. März 1885.

Eine 1/2 Jahr alte Katze. 0,01 Morphium, 0,01 Curare Desinfection mit 5 procentiger Resorcinlösung.

\begin{tabular}{|c|c|c|c|}
\hline & $\begin{array}{l}\text { Luf beiden Manometern } \\
\text { Nach Zurückzie }\end{array}$ & $\begin{array}{r}\text { L } \\
\text { Vordruck } \\
\text { ung des S }\end{array}$ & $\begin{array}{l}\text { inkes Auge. } \\
=32 \mathrm{Hgmm} \text {. } \\
\text { tilets: }\end{array}$ \\
\hline Zeit & & Zeit & \\
\hline $11 \mathrm{~h} 20 \mathrm{~m}$ & $\begin{array}{l}\text { Communication ganz } \\
\text { frei } \quad 28 \mathrm{Hgmm} \text {. }\end{array}$ & & $\begin{array}{l}\text { Communication ganz } \\
\text { frei. }\end{array}$ \\
\hline $\begin{array}{l}11 \mathrm{~h} 25 \mathrm{~m} \\
11 \mathrm{~h} 30 \mathrm{~m}\end{array}$ & Pup. mittelw. 34 & $11 \mathrm{~h} 30 \mathrm{~m}$ & $\begin{array}{l}\text { treI. } \\
\text { pup. } 8 \mathrm{Mm} .36 \mathrm{Hgmm} .\end{array}$ \\
\hline $11 \mathrm{~h} 35 \mathrm{~m}$ & 28 & $11 \mathrm{~h} 35 \mathrm{~m}$ & 36 , \\
\hline $11 \mathrm{~h} 15 \mathrm{~m}$ & $\begin{array}{l}\text { Puls u. Athmungs- } \\
\text { sehwankungen sehr } \\
\text { deutlich } 28 \text { Hgmm. }\end{array}$ & $11 \mathrm{~h} 42 \mathrm{~m}$ & 34 \\
\hline
\end{tabular}




\begin{tabular}{|c|c|c|c|c|c|c|c|}
\hline & $\begin{array}{r}\text { Auf beiden } \\
\text { Nach }\end{array}$ & $\begin{array}{l}\text { ge. } \\
\text { Manor } \\
\text { Zuri }\end{array}$ & etern & $\begin{array}{r}\text { L } \\
\text { Vordruck } \\
\text { hung des } \mathrm{S}\end{array}$ & $\begin{array}{l}\text { inkes Aug } \\
=32 \text { Hgmn } \\
\text { Stilets: }\end{array}$ & & \\
\hline Zeit & & & & Zeit & & & \\
\hline $11 \mathrm{~h} 50 \mathrm{~m}$ & & $26 \mathrm{H}$ & rmm. & $11 \mathrm{~h} 44 \mathrm{~m}$ & $\begin{array}{l}\text { Puls- u. Ath } \\
\text { schwankn }\end{array}$ & $\begin{array}{l}30 \mathrm{I} \\
\text { hmur } \\
\text { nger }\end{array}$ & $\begin{array}{l}\text { grmm. } \\
\text { ss- } \\
\text { sehr }\end{array}$ \\
\hline $11 \mathrm{~h} 55 \mathrm{~m}$ & & 26 & 3 & $11 \mathrm{~h} 53 \mathrm{~m}$ & dentlich & $28 \mathrm{I}$ & $\mathrm{gmm}$. \\
\hline $12 \mathrm{~h}-\mathrm{m}$ & & 26 & $"$ & $12 \mathrm{~b}-\mathrm{m}$ & & 28 & $\eta$ \\
\hline $12 \mathrm{~h} 5 \mathrm{~m}$ & $\begin{array}{c}\text { Atropinin } \\
\text { lation }\end{array}$ & $\begin{array}{l}\text { astal. } \\
26 \mathrm{H}\end{array}$ & $\mathrm{mm}$ & $12 \mathrm{~h} 5 \mathrm{~m}$ & Pup. 8 Mm. & 28 & $"$ \\
\hline $12 \mathrm{~h} 10 \mathrm{~m}$ & Pup. $6 \mathrm{Mm}$ & 26 & 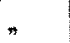 & $12 \mathrm{~h} 12 \mathrm{~m}$ & & 28 & $\Rightarrow$ \\
\hline $12 \mathrm{~h} 20 \mathrm{~m}$ & & 26 & 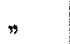 & $12 \mathrm{~h} 20 \mathrm{~m}$ & & 28 & $"$ \\
\hline $12 \mathrm{~h} 25 \mathrm{~m}$ & $\begin{array}{l}\text { Pup. erweit } \\
\text { sich }\end{array}$ & $\begin{array}{l}\text { ert } \\
27\end{array}$ & " & $12 \mathrm{~h} 25 \mathrm{~m}$ & & 28 & $"$ \\
\hline $12 \mathrm{~h} 37 \mathrm{~m}$ & & 25,5 & $n$ & $12 \mathrm{~h} 30 \mathrm{~m}$ & & 28 & $"$ \\
\hline $12 \mathrm{~h} 40 \mathrm{~m}$ & & 26 & 4 & $12 \mathrm{~h} 35 \mathrm{~m}$ & & 28 & $"$ \\
\hline $12 \mathrm{~h} 45 \mathrm{~m}$ & & 26 & $"$ & $12 \mathrm{~h} 42 \mathrm{~m}$ & Pup. $8 \mathrm{MIm}$. & 27 & $"$ \\
\hline $12 \mathrm{~h} 50 \mathrm{~m}$ & $\begin{array}{c}\text { Atropin- } \\
\text { gabe }\end{array}$ & 26 & $"$ & $12 \mathrm{~h} 50 \mathrm{~m}$ & & 26 & $"$ \\
\hline $12 \mathrm{~h} 55 \mathrm{~m}$ & & 26 & $"$ & $12 \mathrm{~h} 55 \mathrm{~m}$ & & 26 & " \\
\hline $12 \mathrm{~h} 57 \mathrm{~m}$ & & 24 & $"$ & $1 \mathrm{~h} \quad 0 \mathrm{~m}$ & & 26 & $"$ \\
\hline $1 \mathrm{~h}-\mathrm{m}$ & & 24 & , & $1 \mathrm{~h} 10 \mathrm{~m}$ & & 26 & $"$ \\
\hline $1 \mathrm{~h} 5 \mathrm{~m}$ & & 24 & " & $1 \mathrm{~h} 15 \mathrm{~m}$ & & 26 & $n$ \\
\hline $1 \mathrm{~h} 10 \mathrm{~m}$ & Pup. 8 Mm. & & 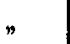 & $1 \mathrm{~h} 20 \mathrm{~m}$ & & 26 & $"$ \\
\hline $1 \mathrm{~h} 13 \mathrm{~m}$ & & 22 & $"$ & $1 \mathrm{~h} 28 \mathrm{~m}$ & & 26 & $n$ \\
\hline $1 \mathrm{~h} 21 \mathrm{~m}$ & & 20 & 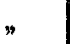 & $1 \mathrm{~h} 27 \mathrm{~m}$ & & 26 & $"$ \\
\hline $\begin{array}{l}1 \mathrm{~h} 25 \mathrm{~m} \\
1 \mathrm{~h} 30 \mathrm{~m}\end{array}$ & Pup. 11 Mm. & $\begin{array}{l}20 \\
20\end{array}$ & " & $1 \mathrm{~h} 30 \mathrm{~m}$ & & 26 & $"$ \\
\hline
\end{tabular}

Keine Puls- und Athmungsschwankungen mehr.

Allgemeines Sinken des Drucks auf beiden Augen:

$1 \mathrm{~h} 40 \mathrm{~m}|\quad 17 \mathrm{Hgmm}| .1 \mathrm{~h} 40 \mathrm{~m} \mid \quad 19,5 \mathrm{Hgmm}$.

Umstehend die zugehörige Curve.

An diesem Versuch sieht man wiederum eclatanter Weise die Druckherabsetzung des Atropins, im ganzen um $6 \mathrm{Hg}-\mathrm{Mm}$.; im Anfang findet sich 20 Minuten nach der ersten Atropin-Einträufelung eine Steigerung ron einem Quecksilbermillimeter, die auf Rechnung des Atropins geschrieben werden kann, für welche wir aber die Erklärung schuldig bleiben müssen, da von Seite des 
Versuchsthieres nichts bemerkbar war, was auch das Manometer des nicht vergifteten Auges durch seinen ruhigen Stand auf $28 \mathrm{Mm}$. notirt.

Ich habe diese primäre, geringe Steigerung des Drucks nur in einem Experimente gesehen, ein Hauptgrund. weshalb ich dessen Protokoll in extenso anführte. So stellen mich denn die Versuche, die ich mit Atropin an curarisirten Katzen ausgeführt und die nach langer

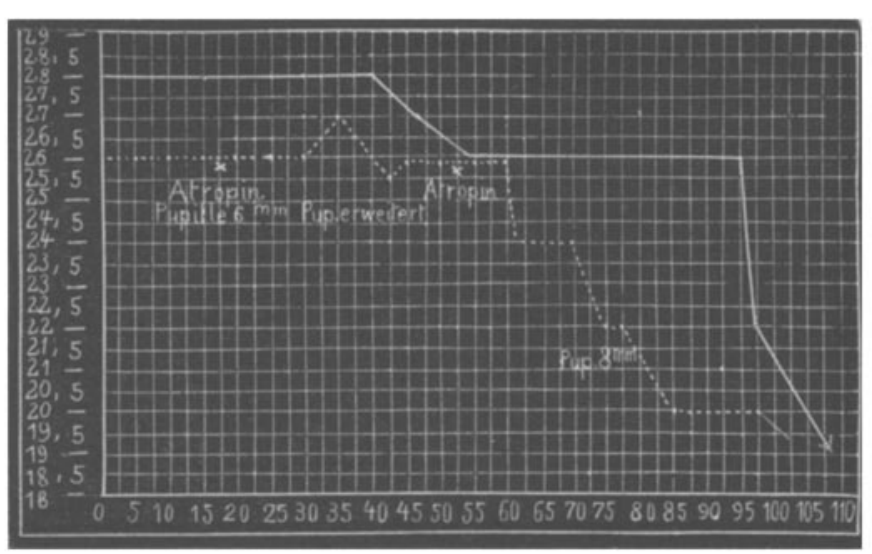

Die Druckhöhen haben auf beiden Augen setr geschwankt whrend 20 bis 30 Minuten, dann erst wurden sie stationär und in dieser Zeit beginnt die Curve.

Figur 2.

Uebung, wie ich glaube mit aller möglichen Sorgfalt und Genauigkeit eingeleitet und verfolgt wurden, in direkten Gegensatz zu den Behauptungen ron Höltzke-Graser, welche nie eine Druckverminderung durch Atropin gesehen haben wollen. Ioh bin durch das, was ich beobachtet habe, gezwungen zu sagen:

Atropin hat im Ange eines curarisirten Thieres nie den Druck gesteigert mit Ausnahme der vorübergehenden Erbơhung im oben angefuhrten Versuch.

Nachfolgende Curve von Atropinwirkung hat Pflüger 
Ueber den Einfluss der Mydriatica und Myotica ete. 133

in Mailand demonstrirt, im Congressberichte kam dieselbe jedoch nicht zum Abdruck. Vide Fig. 3.

In der Höltzke-Graser'schen Versuchsanordnung sind namentlich zwei schwache Punkte, die wahrscheinlich in ursächlichem Zusammenhang stehen mit den Resultaten, welche diese Beobachter mit Atropin gehabt haben. Ich

Graues Kaninchey, curarisint.

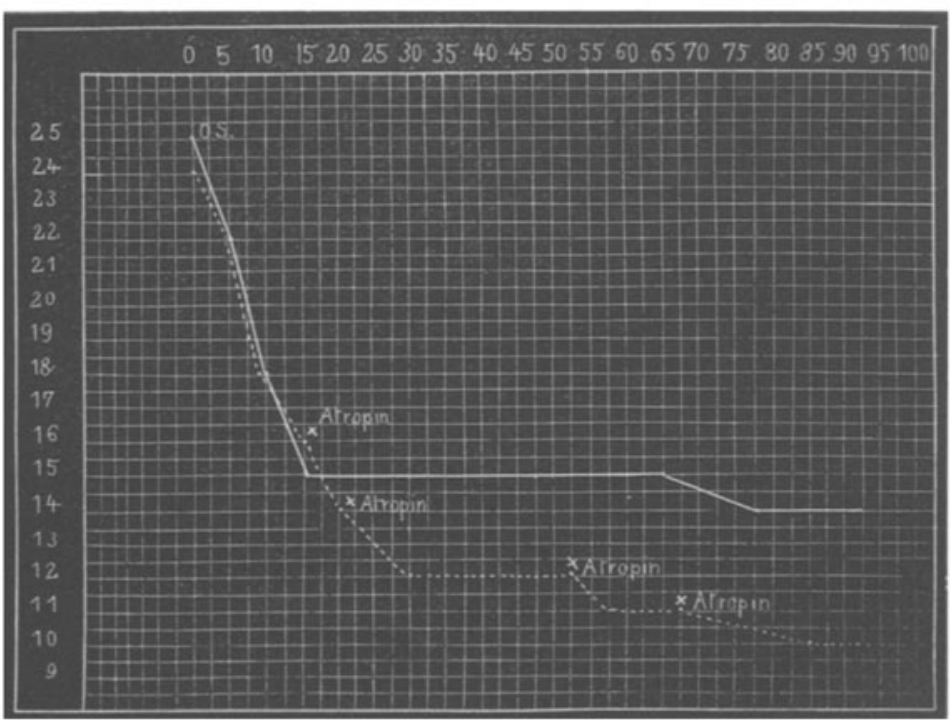

Der Versuch wird unterbrochen, indem das rechte Axge der Kathodenwirkung ansgesetzt wird.

Figur 3.

meine in erster Linie die Thatsache, dass weder Höltzke noch Graser einen Versuch gebracht haben, der zugleich den Anforderungen eines Controlexperimentes entspricht. Obgleich Graser in der Einleitung seiner Arbeit pag. 345 und später pag. 350, exemplificirend mit einem Versuch vom 10. October, davon spricht, wie nothwendig die Controlirung des einen Auges durch das andere sei, 
finden wir in seinen Protokollen nirgends neben dem durch Medikament beeinflussten Auge das andere rein als physiologisches beobachtet. In dem als Schema vorangestellten Versuch, pag. 348 wird zuerst das linke Auge von $4 \mathrm{~h} 5 \mathrm{~m}$ bis $5 \mathrm{~h}-\mathrm{m}$ unter Atropineinwirknng beobachtet, der Versuch nachher abgebrochen; sodann am nämlichen Thiere das rechte Ange mit Eserin behandelt von $5 \mathrm{~h} 25 \mathrm{~m}$ bis $6 \mathrm{~h} 30 \mathrm{~m}$. Dass hierbei keine Controle geherrscht haben kann, levchtet ein. Wer verbürgt mir aber, dass von $4 \mathrm{~h}$ bis $5 \mathrm{~h}$ der Druck in beiden Augen wahrscheinlich mit dem Blutdruck nicht gesunken ist und die halbstündige (links beobachtete) Steigerung keine zufällige, sondern wirklich eine von Atropin herrührende sei. Wenn auch nichts beweisen, so würde für diesen allfülligen Einwand doch sprechen, dass der Anfangsdruck beim linken Auge $28 \mathrm{Mm}$. Hg, beim rechten nach einer Stunde und zwanzig Minuten nur mehr $24 \mathrm{Mm}$. betrug.

Eben so wenig kann von einer Controlirung des einen Auges durch das andere die Rede sein, wenn auf beiden Seiten die Wirkung eines Medikaments auf den intraocularen Druck geprüft wird - und das haben Höltzke und Graser meistentheils gethan. Wenn zum Beispiel die Eserinseite hinab-, die Atropinseite hinaufgeht, so ist doch gar kein zwingender Grund vorhanden, zu sagen: ergo setzt Eserin den Druek herab, Atropin denselben hinauf.

Ein fernerer Grund, weshalb die Höltzke-Graser'sehen Versuche so andere Resultate lieferten als die unserigen in Bezug auf die Wirkung von Atropin, liegt vielleicht in dem Umstand, dass sie nur selten Curare mit künstlicher Athmung zur Narkose benutzten, sondern diese mit Morphium, Chloroform-Aethermischung, Chloral zu erzielen suchten.

Es ist sehr gut möglich, dass gerade, weil die physiologischen oder die durch die Narcotica bewirkten Druck- 
schwankungen in Ermangelung der Controle des andern Auges nicht zum Ausdruck gelangen konnten, und so Steigerungen der Tension in Folge von Exitationsstadien auf Rechnung eines Medikaments geschrieben wurden. Es wäre renn allerdings ein eigenthümliches Zufallsspiel gewesen, hätten immer solche Erregungszustände zeitlich mit den Atropin-Einträufelungen coincidirt; jedoch in den Bereich der Möglichkeit gehört es immerhin. Wir haben es bei unserer sehr ruhigen Curarenarkose gesehen, dass die geringste Zuckung, welche hie und da eintrat, wenn die Wirkung des Gifts aufzuhören drohte, den Druck um einige $\mathrm{Hg}-\mathrm{Mm}$. hinaufzutreiben im Stande war; dass bei Morphium- und Chloroformnarkosen Variationen des Blutdruekes viel häufiger sind, ist bekannt.

Fassen wir in kurzen Worten zusammen, was wir über Atropinwirkung zu berichten haben, so kann dies lauten: Atropin setzt unter physiologischen Verhältnissen, das heisst im Auge des curarisirten Thieres den intraculären Druck langsam herab.

Da Pflüger in einer grossen Reihe von Experimenten am curarisirten und nicht curarisirten Kaninchen stets eine Druckherabsetzung durch Atropin gesehen hat und nie eine Drucksteigerung, so erscheint der Schluss berechtigt: Atropin setzt unter physiologischen Verhältnissen überhaupt den Druck herab.

\section{Cocaïn.}

Dieses Alcaloid der Cocablätter, von der Ostseite der Sudamerikanischen Anden stammend, wurde erst nach den Mittheilungen von C. Koller *) in Wien über seine local anästhesirende Wirkung aufs Auge von den Ophthalmologen berücksichtigt, dafür aber mit dem Sturm der Begeisterung,

*) Wiener medizin. Wochensehrift, November 1884. 
der nur einem Medikament zukümmt, das eine dominirende Stelle im Arzneimittelschatz einzunehmen berufen ist.

Ueber die Eigenschaften dieser neuen Drogue ist unter vielen andern ron Ad. Weber*), Holtzke*), Emmert**) gesprochen und geschrieben worden. Der Einfluss auf die Nervenendigungen der Schleimbäute, auf die Accommodation, auf die äussern Augenmuskeln, der reizende Einfluss auf den N. sympathicus sind in den versehiedenen Arbeiten besprochen worden. Hier interessirt uns nur der Einfluss des Alcaloid auf den intraoculären Druck in seiner Eigenschaft als Mydriaticum. Was das betrifft, hat Ad. Weber in seiner oben angefuhrten Arbeit gefunden, dass Cocaïn im ,normalen sowohl als entzündlich resistenteren Auge" eine dentliche Herabsetzung der Corneal- sowie Scleralspannung bewirke.

Die Dauer der Cocaïnmydriase geben einige auf eine halbe Stunde, andere bis auf zwei Stunden an. Die Pupillenerweiterung geht nicht wie bei Atropin gleichmässig von statten, sondern geschieht excentrisch oder unregelmässig. Die Mydriase soll bei stark pigmentirten Leuten beträchtlicher zu Stande kommen als bei schwach pigmentirten Individuen.

Die pupillenerweiternde Eigenschaft des Cocaïn hat mich bestimmt, diesen Körper ebenfalls in den Bereich der manometrischen Augendruckmessungen zu ziehen, um so mehr als so viel ich weiss bis jetzt dieses Alcaloid noch nicht in dieser Weise untersucht worden ist. Ad. Weber hat seine obige Behauptung auf Grund tonometrischer Erfahrungen aufgestellt. Ueber die Dauer der Mydriase können wir sagen, dass sie nach 40, 50 Minuten bis einer Stunde längstens vorüber war.

Wir benutzten zur Instillation eine Lösung von $4 \%$

*) Klinische Monatsblätter für Augenheilkunde. XXII. Jah"gang December. pag. 443 u. folg.

* Correspondenzblatt für Schweizer Aerzte, XV. Jg. No. 6, pag. 129, u. folg. 
Ueber den Einfluss der Mydriatica und Myotica etc. 137

Cocaïn. muriat. - Es sei uns auch hier gestattet, an Hand zweier Versuchsprotokolle mit zugehörigen Curven das klar zu machen, was wir nach unsern Experimenten über die Wirkung dieses Medikaments auf den Augendruck zu sagen berechtigt sind.

XXI. Versuch. 22. A pril 1885.

Ausgewachsene Katze. 0,01 Morph. tracheotom., 0,01 Curare. $9 \mathrm{l} 15 \mathrm{~m}$ Ueberdruck in beiden Manometern $30 \mathrm{Hgmm}$.

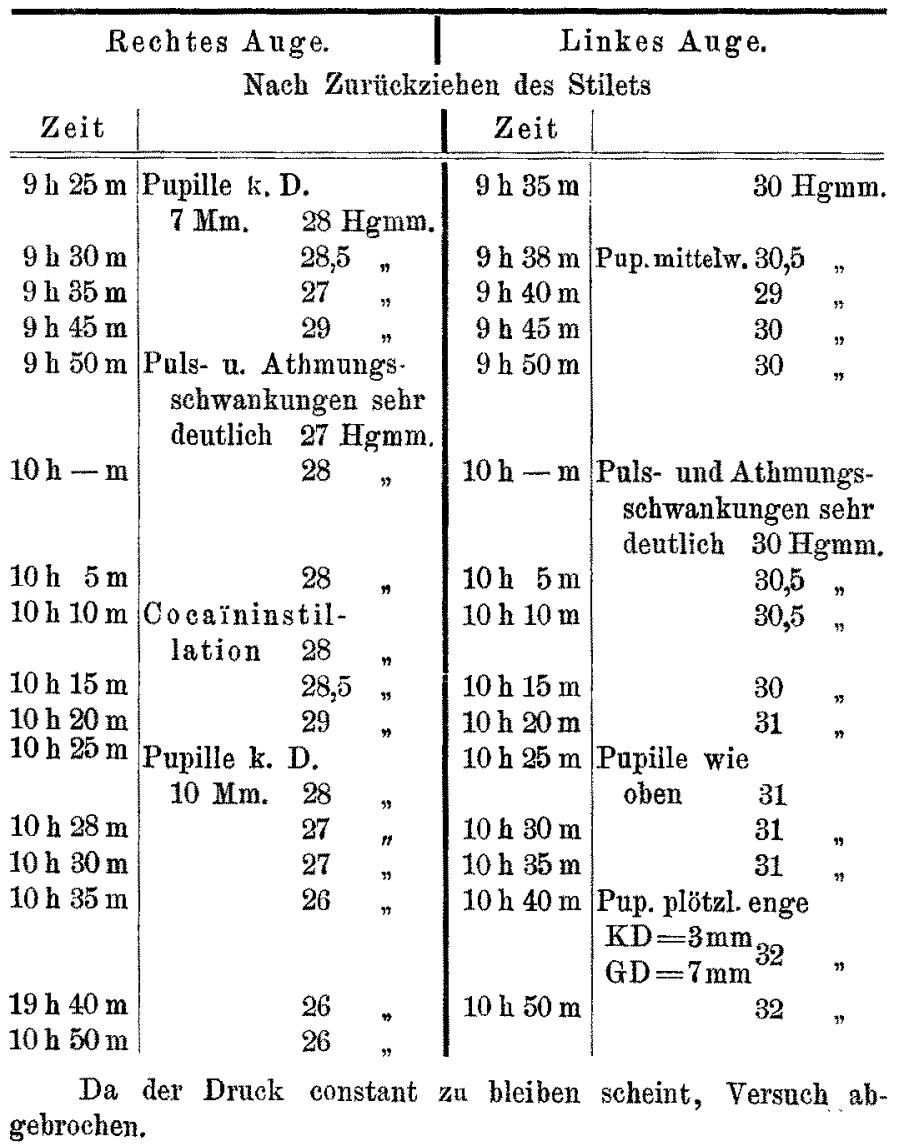


Bei Betrachtung nachstehender Curvenzeichnung (Fig. 4) sehen wir, dass neben einem unruhigen, zur Hohe tendirenden physiologischen Druck (weiss ausgezogene Linie) die Augenspannung auf dem rechten Auge gleich nach der Einträufelung von Cocaïn innerhalb 10 Minuten um einen Millimeter steigt, um in den nächsten fünf Minuten auf den Anfangspunkt zurückzngehen. In den nächsten 10 Minuten sinlt die Cocaïncurve in zwei $\mathrm{Ab}$ stufungen um zwei Millimeter, um dort constant zu bleiben. Interessant ist an diesem Versuch ferner, dass während

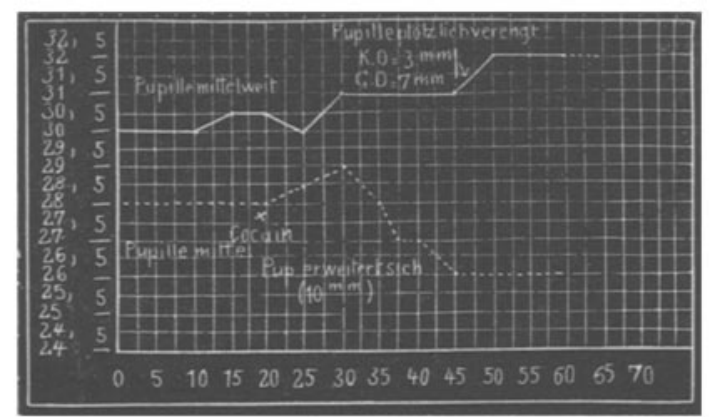

Figur 4.

dem der physiologische Druck seine Culmination erreicht, die Pupille des betreffenden Auges beinahe plötzlich sehr enge wird, nachdem sie 12 Minuten vorher noch mittelweit gewesen. Für diese Myose wissen wir keinen Grund anzugeben. Das Thier war ganz normal, die Einführung der Canüle ins Auge vollkommen gelungen, die Iris nicht gestreift.

Die primäre kleine Drucksteigerung haben wir bei der Mehrzahl der Cocaïnversuche gesehen, sie macht aber bald einer erheblichern Druckerniedrigung Platz. Letztere betrug immer 2 bis 3 Millimeter Quecksilber. In zwei Versuchen sahen wir den Druck ohne Steigerung sinken, aber auch da blieb es bei den kleinen Zahlen. Wir wollen 
Ueber den Einfluss der Mydriatica und Myotica ete. 139

einen einschlägigen Versuch in extenso anführen, indem wir betonen, dass dieser einzelne Abweichungen von der typischen Cocaïnwirkung repräsentirt. Hinter dem Versuchsprotokoll lassen wir wiederum die entsprechende Curvenzeichnung folgen.

XXIV. Versueh. 25. April 1885.

Mittelgrosse Katze. 0,01 Morph. subeut., 0,01 Curare. $9 \mathrm{~h} 5 \mathrm{~m}$ Ueberdruck beidseitig $30 \mathrm{Hgmm}$.

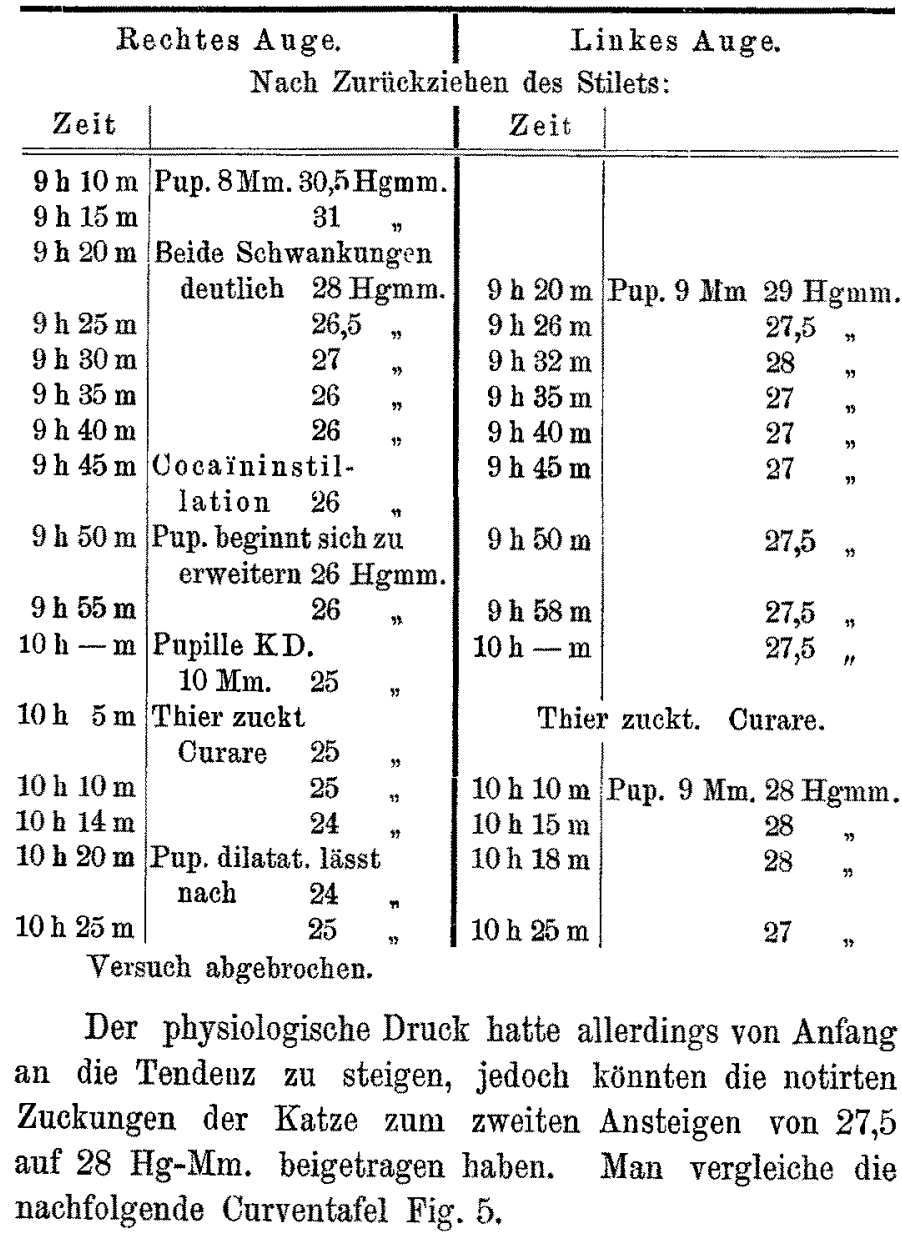


Unsere Resultate, die wir aus den Cocaïnexperimenten gewonnen haben, heissen:

I. Cocaïn setzt den intraoculären Druck unter normalen Verhältnissen herab, ungefähr um 2 bis 3 Millimeter.

II. Cosaïn schickt in der Mehrzahl der Fälle der Verringerung der Augenspannung eine kleine Erhöhung derselben voraus.

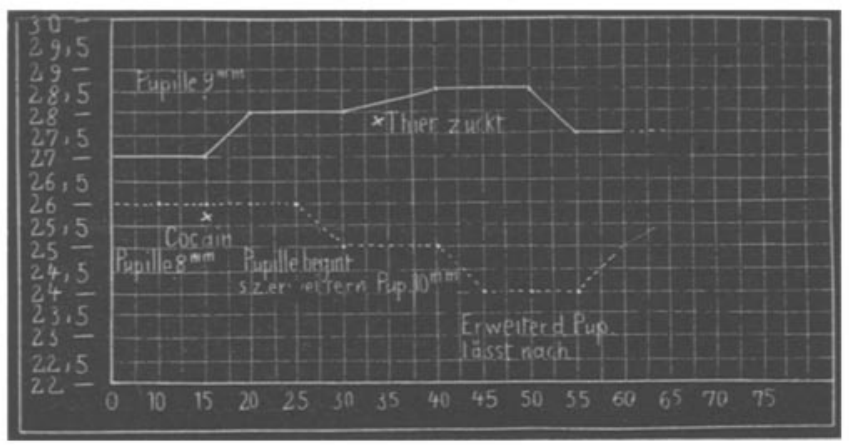

Kigux ö.

Die kleine, primäre Erhöhung des Drucks dürfte vielleicht auf einer vorübergehenden Reizung der Secretionsnerven des Auges beruhen.

\section{Eserin.}

Zur Einträufelung benutzten wir eine 1procentige Lösuñg von Eserinum sulphuricum. Bei unsern frühern Kaninchenversuchen brachten wir nie eine schöne Myose zu Stande. Der Sphinkter Iridis dieser Thiere muss bedeutend schwächer entwickelt sein als derjenige der Katzen, wo man bei vollkommener Myose die Pupille als feines, schwarzes, senkrecht gestelltes Strichelchen wahrnimmt. Aus dem Versuchsprotokoll ersieht man leicht, wie das Eserin in unsern Experimenten den intraoculären Druck 
Ueber den Einfluss der Mydriatica und Myotica etc. 141 beeinflusste. Der Versuch, dessen Protokoll wir hier mittheilen, kann als repräsentirender Typus der Mehrzahl der Eserinversuche betrachtet werden.

XVII. Versuch, 20. März 1885.

Alte Katze. 0,01 Morphium mur. subcut. 0,01 Curare. $9 \mathrm{~h} 55 \mathrm{~m}$ Ueberdruck in beiden Manometern $32 \mathrm{Hgmm}$.

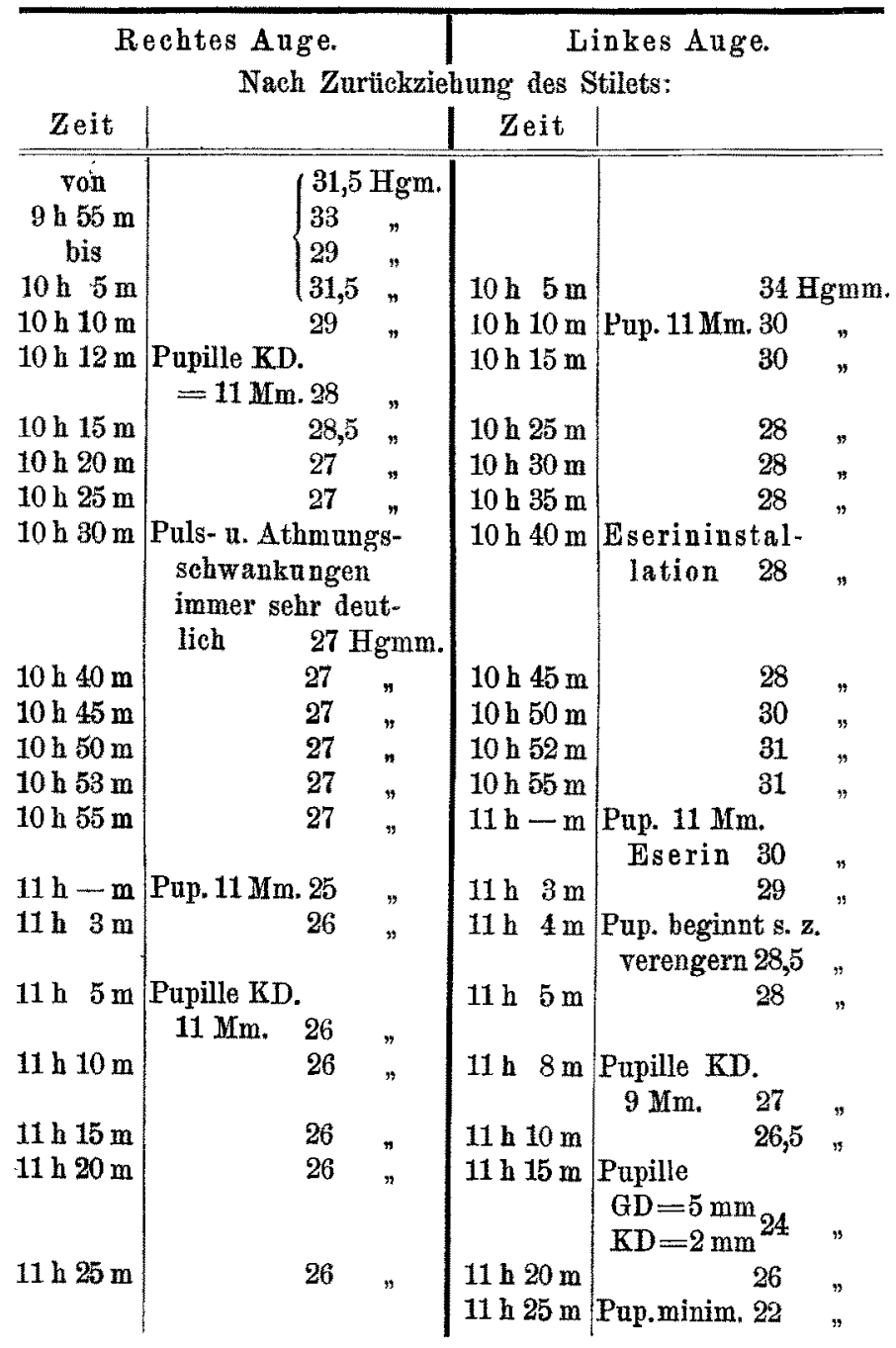


Wir haben also hier (vergl. Curventafel, Fig. 6) nach der Finwirkung des Eserins eine Drueksteigerung, welehe $3 \mathrm{Mm}$. beträgt, nach 20 Minuten wiederum zum früheren Stand zurückkehrt, dann aber einer erheblichen Druckherabsetzung Platz macht. Diese geht, wie der punktirte Curvenschenkel zeigt, ziemlich rasch abwärts und erreicht, nachdem sie noch eimmal einen plötzlichen Sprung von 2 Millimeter aufwärts gethan hat, innerhalb 20 Minuten

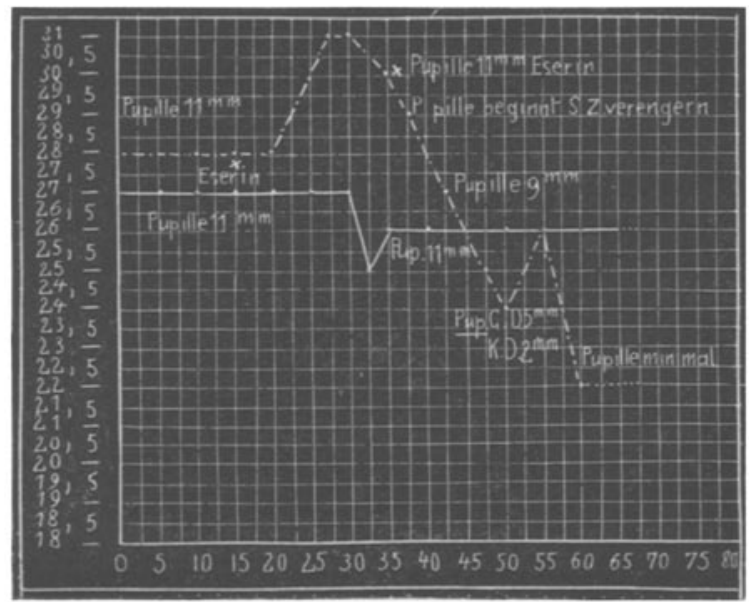

Figur 6.

ihr Minimum, wo sie längere Zeit zu verharren scheint. Wir sehen demnach eine primäre Druckerhöhung von 3 Millimetern und eine nachfolgende Druckherabsetzung unter den Anfangsdruck, während die Druckböhe im physiologischen Auge nach einer plotzlichen Schwankung nach unten die letzte halbe Stunde 1 Millimeter tiefer stehen bleibt als anfangs (Anfangs $27 \mathrm{Hg}-\mathrm{Mm}$., die letate halbe Stunde $26 \mathrm{Hg}-\mathrm{Mm}$.)

Was die Pupillenweite anbelangt, so beginnt die Myose erst, nachdem die Curve den höchsten Punkt schon 
Ueber den Einfluss der Mydriatica nud Hyotica etc. 143

überschritten hat und erreicht im Druckminimum ihr Maximum. Die physiologische Curve bewegt sich verhältnissmässig sehr ruhig in diesem Versuche. Nachdem die Schwankungen, vom Einstich der Canüle herrührend, sich beruhigt haben, steht der Druck zuerst während 30 Minuten auf $27 \mathrm{Hg}-\mathrm{Mm}$. Mit einer wälrend 5 Minuten verlaufenden Excursion nach abwärts verbleibt er continuirlich auf $26 \mathrm{Hg}-\mathrm{Mm}$. Die Pupille blieb sich auf dem rechten Auge stets gleich.

Unsere Versuche mit Eserin, wovon der aufgeführte, wie schon bemerkt, einen wahren Typus repräsentirt, haben in erster Linie eine Drucksteigerung notirt, die sich sehr bald nach der Einträufelung des Myoticums geltend macht. Dies haben Höltzke-Graser und auch Pflüger schon constatirt. Letzterer konnte zwar, weil er mit Kaninchen experimentirte, die darauffolgende Druckherabsetzung nicht constatiren, da ihm der Versuch jedesmal durch plastische Iritis verdorben wurde, deren Exsudat die Canüle verstopfte.

In unsern Versuchen war die secundäre Drnckherabsetzung stärker als die primäre Druckerböhung; im obigen Versuche haben wir $3 \mathrm{Hg}-\mathrm{Mm}$. Erhöhung und nachher $4 \mathrm{Hg}-\mathrm{Mm}$. Herabsetzung unter den ursprünglichen Stand. In den Protokollen finden wir einen einzigen Versuch, der in der Herabsetzung des Drucks einen HgMillimeter weniger notirt als bei der Steigerung. Dieses Experiment unterscheidet sich auch von den übrigen einschlägigen dadurch, dass der absteigende Curvenschenkel der primären Druckerhöbung bedeutend flacher (10 bis 20 Minuten) verläuft als derjenige der andern Eserincurven (vide nachstehende Fig. 7).

Fassen wir die Resultate unserer Experimente zusammen, so müssen wir von der Wirkung unseres Calabaralkaloides auf den Intraoculardruck sagen:

I. Eserin erhöht primär den Druckim physio- 
logischen Auge. Eine primäre Herabsetzung der Tension kommt nicht vor.

II. Eserin setzt nach der Erhöhung die intraoculäre Spannung quantitativ mehr herab als es sie primär steigerte.

Die Zeit der primären Drucksteigerung entspricht dem Auftreten der irradirenden Ciliarschmerzen, die Eserin am Menschen oft verursacht. Es dürften diese beiden

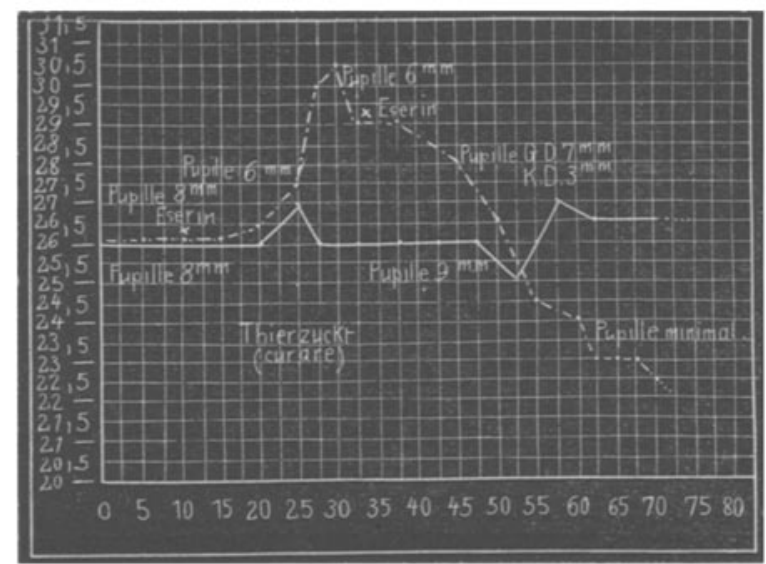

Fĭgur 7.

Fakta auf Trigeminuswirkung zurückzuführen sein; da der Trigeminus zugleich Secretionsnerv fürs Auge ist, liesse sich die primäre Tensionsvermehrung ebenfalls auf Reizung desselben zurückleiten. Ebenso erklärlich wäre dann die primär druckherabsetzende Wirkung des Atropins, welches als Narkoticum wirken würde. Ist es doch bekannt, dass nach schmerzhaften Eingriffen ins Auge eine Atropineintränfelung wohlthuend wirkt.

$$
\text { IV. Pilocarpin. }
$$

Das Alkaloid der Blätter des Pilocarpus pennatifolius ist als Myotieum schon mehrere Jahre bekannt; es wurde 
Ueber den Einfluss der Mydriatica und Myotica ete. 145

zuerst von Pflüger auf seine Wirkung, den intraoculären Druck betreffend, geprüpft. Er hat seine bezulglichen Manometerversuche an narkotisirten Kaninchen gemacht und im Allgemeinen eine langsame Herabsetzung der Druckhöhe bemerkt. Die zweite Untersuchungsreihe über diesen Gegenstand hat Höltzke in der XIII. Sitzung (den 15. Mai 1885) der physiologischen Gesellschaft zu Berlin *) veröffentlicht. Er sagt, dass Pilocarpin ,den Druck in der Kammer ,primär' erhöht."

Das Pilocarpin ist jedoch ein Stoff, der, in den Kreislauf gebracht, das ganze Thier alterirt. Der Puls wird schneller und voller, es tritt Schweiss und Salivation auf. Mit dem Auftreten der letztern kommt das Thier in starke Aufregung. Dies Alles ist der Grund, warum, wie wir sehen werden, die Drackeurve nicht nur des unter Pilocarpinwirkung stehenden Auges, sondern auch der: controlirenden Seite im Anfang hüpfend hinauf- and hinunter steigt.

Wir gaben das Pilocarpin in 2procentiger Lösung tropfenweise in den Conjunctivalsack. Die Salivation trat bald nach der Instillation auf (ungefähr nach 5 bis 10 Minuten.)

Nachfolgende zwei Versuchsprotokolle sind den Curvenbildern 8. und 9. zu Grunde gelegt.

XXIX. Versuch. 23. April 1885.

Mittelgrosse Katze. 0,01 Morph. mur. subcut., Curare 0,01. Ueberdruck auf beiden Augon $28 \mathrm{Hgmm}$.

\begin{tabular}{l|l|l|}
\hline \multicolumn{2}{c|}{ Rechtes Auge. } \\
Nach Zurückziehung des Stilets: \\
Zeit
\end{tabular}

*) vide Verhandlungen der physiolog. Gesellschaft zu Berlin Jahrg 1884 - 85, No. 13.

v. Gragfe's Arehiv für Oplihainologie, XXXmr. 1. 


\begin{tabular}{l} 
Rechtes Auge. \\
Nach Zurückziehung des Stilets: \\
Zeit \\
\hline
\end{tabular}

Puls- und Athmungsschwankungen sind aufgehoben.

Versuch abgebrochen.

Wie aus der Curventafel 8 ersichtlich ist, giebt Pilocarpin circa während 40 Minuten die exquisitesten 
Druckschwankungen, das Quecksilber macht Ausschläge von 4-6 Mm. Die physiologische Curve bleibt jedoch nicht zurück, sie steigt und fällt so ziemlich parallel mit derjenigen des Pilocarpinauges.

Es rührt dies, wie schon oben angedeutet, von der allgemeinen Erregung des Thieres her, und ist in dieser Zeit der Einfluss des Pilocarpins auf den betreffenden Augendruck nicht zu eruiren. Erst nachdem sich das

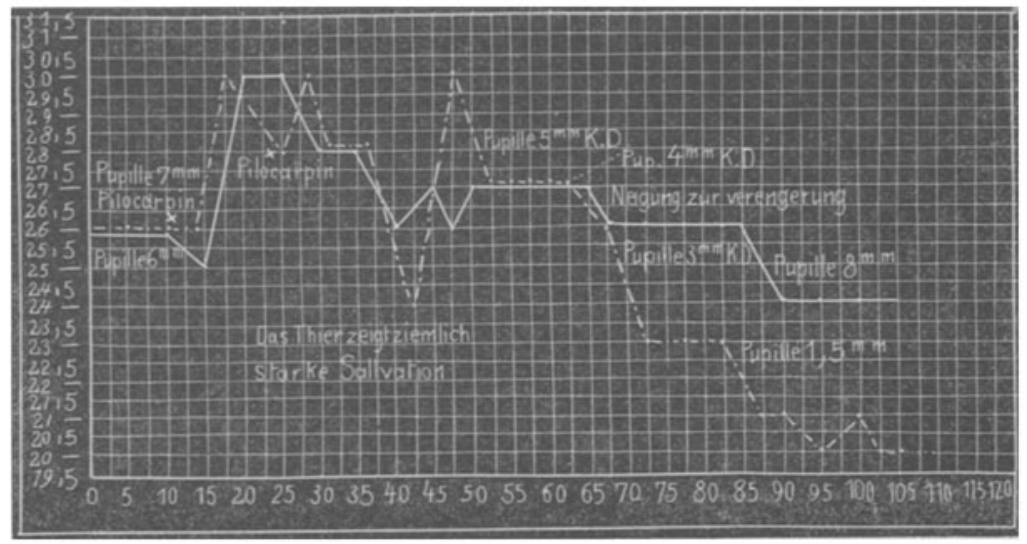

Die Curve beginnt um $9 \mathrm{~h} 13 \mathrm{~m}$.

Figur 8.

Quecksilber auf beiden Seiten etwas beruhigt hat (es stehen beide Drucke während 10 Minuten auf gleicher Höhe), fullt die Pilocarpineurve steil $a b$ und sinkt von nun an bis zum Schluss des Versuchs um $7 \mathrm{Mm}$., während die Herabsenkung des physiologischen Druckes $3 \mathrm{Mm}$. beträgt. Das Sinken des gesammten Druckes ist jedenfalls als Reaction der primären Erregungsschwankungen aufzufassen. Die Zeit dieser letztern war in einigen Fällen kürzer und deshalb lassen wir als Typus der zweiten Halfte der Pilocarpinexperimente den nächsten Versuch folgen. 
XXXIII. Versuch. Katze. 0,01 Morph. subeut., 0,01 Curare. Ueberdruck beiderseits $28 \mathrm{Hgmm}$.

Rechtes Auge.
Nach zurückgezogenem Stilet:
Z eit

Puls- und Athmungsschwankungen werden beinahe unsichtbar. Quecksilber immer auf demselben Stand, deshalb Abbruch des Versuches. 
In diesem Versuche spielt sich die Aufregungsscene so ziemlich innerhalb 25 Minuten ab. Die Pilocarpineurve zeigt am Ende des Versuches eine Erniedrigung des Druckes um 7 Millimeter gegenüber dem Anfangsdruck desselben Anges, und um 5 Millimeter unter den Stand des physiologischen Druckes des andern Auges.

So haben wir denn im Gegensatz zu Pflüger eine ziemlich lange dauernde Aufregung mit bedeutenden

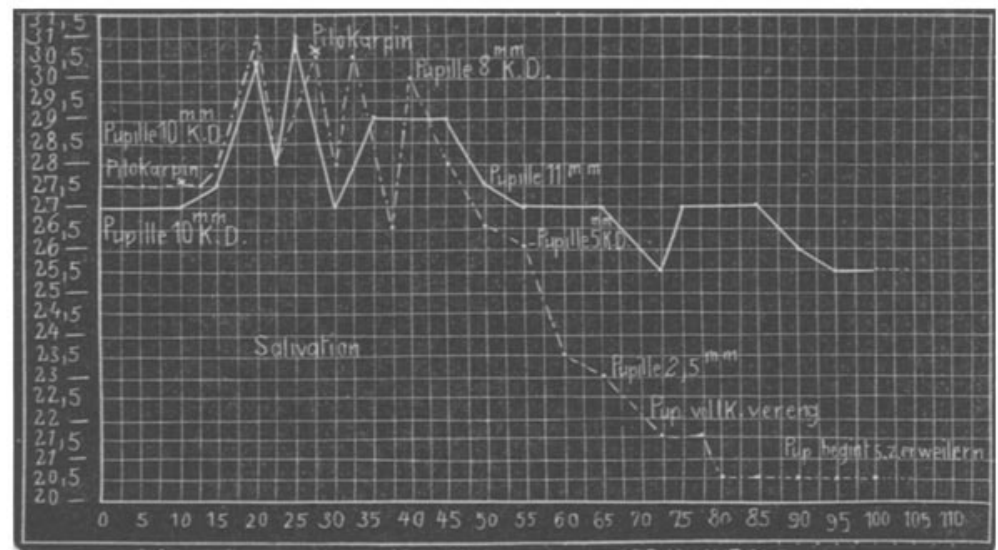

Puls- and Athmungssohwankungen nicht mehr deutlich. Versuch abgebrochen. Die Curve beginat $10 \mathrm{~h} 40 \mathrm{~m}$.

Figur 9.

Druckschwankungen auf beiden Augen gesehen, die dann erst etwa nach 30 Minuten der Druckerniedrigung auf dem Pilocarpinauge Platz machte. Nicht zu vergessen ist, dass Pflüger an Kaninchen experimentirte und es ist möglich, dass diese Thiere weniger auf Pilocarpin reagiren als Katzen und deshalb das Erregungsstadium kürzer ausfiel. Meine Schlusssätze, die Pilocarpinwirkung betreffend, lauten:

1. Pilocarpin setzt im physiologischen Auge den Druck langsam herab, nachdem es 
primär, während circa einer halben Stunde, mit allgemeiner Körperaufregung, die Höhe der Augenspannung beiderseits stark ins Schwanken gebracht hat.

2. In der Periode der Druckschwankungen bemerkt man eine Erhöhung des mittleren Augendrucks auf beiden Seiten.

Aus allen diesen Versuchen, die wir mit den verschiedenen Mydriaticis und Myoticis angestellt haben, ergiebt sich uns nothwendiger Weise folgender Satz:

Das Pupillenspiel steht nicht in nothwendigem, ursächlichem Verhältniss zur Steigerung oder Verminderung des Druckes im Auge.

Es ist dies die Negation der Behauptung von Höltzke und Graser*), welche neuestens von Höltzke in seinem Vortrage in der Berlin. physiolog. Gesellschaft noch einmal vertreten wurde**) mit den Worten: ,3. Im nioht vergifteten Auge (unter physiologischen Verhaltnissen) steigt der Kammerdruck mit Erweiterung und sinkt mit Verengerung der Pupille."

Unsere Behauptung stützt sich in erster Linie auf unsere Atropinversuche, wo bei grösster Mydriase der Druck am kleinsten war, dann gab das Eserin mit zunehmender Myose ebenfalls Verminderung des Drucks. Cocaïn setzte in der Mehrzahl der Fälle, nachdem die Mydriase schon begonnen hatte, die Druckhöhe hinauf, um sie nachher herunterzudrüeken.

Suchen wir nach einer Erklärung der so verschiedenen Resultate unserer Untersuchungen im Gegensatz zu denjenigen von Höltzke-Graser, so könnte folgendes Raisonne-

*) Vide d. oben eitirten Arbeiten.

**) No. 13 d. Verhandl. der physiol. Gesellsch, z. Berlin p. 35. 
ment stichhaltig sein. Neben den, schon oben angeführten Einwänden gegen die Versuchsanordnung der obigen Experimentatoren ist vor allem die physiologische Thatsache zu bern̈cksicbtigen, dass psychische Momente für die Augendrucksteigerung eine grosse Rolle spielen. Abgesehen von den Veränderungen des Blutdruckes tritt bei psychischen Erregungszuständen in jedem nicht immobilisirten (eurarisirten), oder ungenügend narkotisirten Versuchsthiere eine Reflexaction des vasomotorischen Centrums, sowie der Lidmuskulatur, der quergestreiften, äussern Augenmuskeln auf, welche den Augendruck plötzlich in die Höhe zu treiben im Stande ist. Dies hat Pflüger auch bemerkt bei seinen Experimenten ohne Curare, weshalb er verschiedene Vorsichtsmaassregeln gebrauchte, um möglichst wenig Gleichgewichts-Störungen der psychischen Stimmung des Thieres hervorzurufen. Er vermied grelle Beleuchtung, ebenso jede Erregung des Acusticus und der sensiblen Nerven. Er beobachtete, dass z. B. Iautes Auftreten auf dem Fussboden, Fallenlassen eines Gegenstandes, lautes Sprechen oft den Druck plötzlich in positivem Sinne alteriren konnte. Dies alles sahen wir nicht in der ruhigen Curarenarkose. Nachdem sich das Quecksilber nach dem Einstich in die Kammer beruhigt hatte, konnte man den Druck auf dem sog. physiologischen Ange oft über eine Stunde lang auf derselben Hohe stehen sehen, bei vollkommener Erhaltung der Puls- und Athmungsschwankungen. Die nothwendige Folge davon war, dass die betreffenden Curven von $5 \mathrm{zu}$ (meistens) 5 Minuten abgelesen, sehr regelmässige Linien zeigten. Unregelmässig wurde die Curve des physiologischen Auges in unsern Experimenten nur bei den Pilocarpinversuchen, eben aus dem schon oben (vergl, pag. 145) angeführten Grunde, weil das betreffende Alkaloid nicht nur Veränderungen in dem seiner Wirkung als Myoticum unterstellten Auge, sondern eingreifende Stö- 
rungen im ganzen Thiere bewirkt. - Die unregelmässigen Curven von Höltzke und Graser erilären sich also wahrscheinlich zum grossen Theile auch aus der psychischen Aufregung ihrer Versuchsthiere in der ungenügenden Narkose,

Nachdem ich die Resultate meiner Experimente mit manometrischer Druckmessungsmethode erörtert habe, will ich nur in kurzen Zügen einer Versuchsreihe Erwähnung than, die ich ebenfalls anf dem Bernischen physiologischen Institute während des Winters 1884/85 gemacht habe. So präcis an und für sich die manometrischen Messungen des intraocularen Druckes sind, insofern nämlich das nicht vergiftete Auge mit dem andern beobachtet wird, so macht sich doch ein Bedürfniss geltend, die Methode in dieser Richtung zu verbessern. Ideal müsste es sein, einem Auge, dass der Wirkung eines Mydriaticum oder Myoticum unterstellt ist, gewissermaassen anzusehen, wie sich die Spannung in demselben verhalten möchte; ich meine, dass man dahin käme aus andern physikalischen Eigenschaften der Bulbusoberfläche, auf die

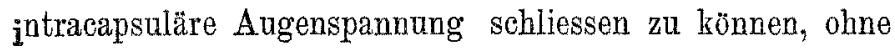
das Auge während des Experiments irgendwie durch Stich oder Druck zu alteriren.

Dies könnte dadurch geschehen, dass man die Krümmungsoberfläche der einer Messung zugänglichen Hornhaut in Relation bringt zum intraoculären Druck in der Weise, dass man verlangt, der Bulbus als eine mit Flüssigkeit gefüllte Blase müsse, wern sein Innendruck steigt, bestrebt sein, Kugelgestalt anzunehmen. Dies vorausgesetzt, muss sich die Cornea, als ein der schwächer gekrümmten Sclera aufgesetztes, stärker gekrümmtes Hohlkugelsegment bei Aenderungen des Binnendrucks, namentlich am Limbus, verändem. Dieser muss bei Steigerung 
der intraoculären Spannung sich abflachen, bei Druckherabsetzung noch mehr eingezogen werden, wobei es sich dann jedenfalls fragt, wie gross die Starrheit der betreffenden Augenhäute sei.

Helmholtz, der Erfinder der Messung der Augenmedien, hat in seiner physiologischen Optik*) angefuhrt, dass die Accommodation die Krümmung der Hornhaut nicht ändert. In seiner Arbeit über die Accommodation des Auges**) sagt er, dass im Allgemeinen die Höhe des intraocularen Drucks einen Einfluss ausube auf die Länge des Krümmungsradius der Hornhant, und vindicirt in dieser Beziehung dem Ophthalmometer eine Zakunft als Spannungsmesser der Augenkapsel. An exstirpirten Kaninchen- und Menschenaugen hat Schelske***) in dieser Richtung Versuche angestellt, indem er in dieselben ein bestimmtes Quantum Flüssigkeit eintrieb und so den Druck erhöhte. Die ophthalmometrischen Messungen vor und nach der Injection zeigten, dass die Cornea sich abflachte, respective der Krümmungsradius länger wurde.

Zu mehr oder weniger negativen Resultaten kamen Donders $\dagger$ ) and Coccius. $\dagger$ ) Sie konnten keinen Unterschied bemerken bei Vergleichung eines glaucomatösen Auges mit dem gesunden des betreffenden Individuums. Coccius fand sogar bei Kranken mit herabgesetztem Augendruck einen längern Krümmungsradius als auf der gesunden Seite, und dies in der Mehrzahl der Fälle.

Mautbner + ) sah an einem Auge mit quellender

*) pag. 120 .

**) Archī für Ophthalmologie I. 2.

*** Archir für Ophthalmologie. X. 2.

†) A. f. Ophthalm. VIII. 2. p. 163.

+) Ophthalmometrie und Spannungsmessung am kranken Auge. Leipzig 1872.

tit) Vorlesungen über die opt. Fehler des Auges. Wien 1876. pag. 222 . 
Cataracta traumatica den Hornhautradius nach Entfernung des Staars um 1,8 Millimeter zurẗckgehen. Woinow*) bemerkte an zwei Migränekranken während des Anfalls eine Verlängerung des Krümmungsradius um ca. 0,4 Mm.

Umfangreiche Cornealmessungen machte $v$. Reuss in seinen Experimenten "Ueber die Wirkung des Eserins auf das normale Auge." **) Er untersuchte die Wirkung des Eserins auf die Krümmung der Hornhaut. Durch eine sinnreiche Aufstellnng brachte er es daza, gleichzeitig mit den Veränderungen der Hornhaut den Gang des Accommodationskrampfes zu beobachten, der jedesmal bei Eserinisirung anftritt. Er fand Verkürzung des Radius durch Eserin von 0,04. bis 0,14 Mm. Die Einwirkung anf die Accommodation findet früher statt als die auf die Hornhautwölbung. Als Dentung dieser Daten: Die Contraction des Ciliarmuskels übt einen Einfluss auf die Hornhautwolbung im oben angegebenen Sinne aus. Ob die stärkere Hornhautwölbung als Ausdruck des verminderten Druckes in der vorderen Augenkammer anzusehen sei, ob sich also das Ophthalmometer als Spannungsmesser bewährt habe, wagt $\nabla$. Reuss noch nicht zu beantworten.

Mit der manometrischen Untersuchung unserer vier Medikamente beschäftigt, wollte ich den Einfluss derselben auf die Hornhautkrümmung ebenfalls mit in Beobachtung ziehen, um dann durch Vergleich beider Resultate heraus zu bekommen, ob wirkliche Druckerhöhung und Druckerniedrigung, wie sie das Manometer zeigte, Hand in Hand gingen mit Verlängerung und Verkürzung des Hornhautradius. Da mir jedoch zur Vollendung dieser zweiten Reihe von Untersuchungen die Zeit fehlte, will ich in diesem Anhang zu meiner Arbeit nur kurz meine

*) Ophthalmometrie. Wien 1s71. p. 68 .

* Archiv fur Ophthalmologie XXII. 3. 
Ueber den Einfluss der Mrydriatica und Myotiea etc. 155

Resultate, betreffend die Beeinflussung des Hornhautradius durch Mydriatica und Myotica, wiedergeben.

Die Instrumente und Lokalitäten wurden mir von Herrn Prof. Dr. Kronecker in liebenswürdigster Weise zur Verfügung gestellt. Als Versuchsthiere benutzte ich Kaninchen. Die Aufstellung der Apparate war folgende:

An der einen Schmalseite eines rechteckigen Tisches ward auf 2 Stativen eine Gasröhre aufgeschranbt, die mit

Ophthalmoskop.

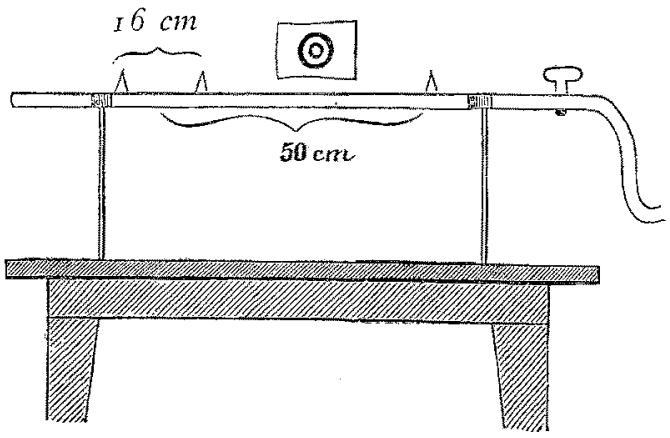

Figur 10.

der Gasleitung des Institnts durch einen Gummischlauch in Verbindung gesetzt werden konnte. Diese Gasröhre ist, an 3 Stellen angebohrt, mit Spitzbrennern versehen. Die Entfernung des einen Brenners von der Mitte des Abstandes der beiden andern beträgt genau $50 \mathrm{Cm}$. Die Distanz der letztern beträgt $16 \mathrm{Cm}$. Direct gegenüber dieser Gasröhre, am gegenüberliegenden Tischende, ist ein Brett senkrecht befestigt; dieses hat in der Höhe des bei der Röhre aufgestellten Ophthalmometers ein Augenloch (wir nennen es so, weil das Auge des betreffenden Versuchsthieres immer genan in dieses Loch eingestellt wurde.) Die Mitte dieser Oeffnung ist von der Mitte der Gasröhre genau $100 \mathrm{Cm}$. entfernt. Also hatte man Grösse des 
Gegenstandes $=50 \mathrm{Cm}$, Entfernung des Gegenstandes $100 \mathrm{Cm}$.

Das Kaninchen wurde in einen Kasten gesetzt, aus welchem nur sein Kopf herausragen konnte; das Thier wurde weder narkotisirt noch curarisirt. Nach einigen vergeblichen Anstrengungen, sich aus dieser Klammer zu befreien und den Kopf zurückzuziehen, verhielten sich die meisten Thiere während des ganzen Versuchs vollkommen ruhig, indem sie sich bald an die garnicht sehr unbequeme Lage gewöhnten. Beistehend ein Schema von einem in den Kasten gesetzten Kaninchen.

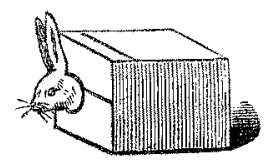

Figur 11.

Der Kasten wurde nun sammt dem Thier so an das Brett auf dem Versuchstisch gebracht, dass das zu untersuchende Ange in die Mitte des Augenloches zu liegen kam. Der Kopf des Kaninchens wurde in einigen Versuchen mit dem Kopfstück eines Kaninchenhalters an ein Stativ befestigt, welches neben dem Augenbrett auf den Tisch geschraubt war.

Díe ophthalmometrischen Messungen wurden an beiden Augen vorgenommen, das eine Auge bildete jedoch auch hier wie in den Manometerversuchen immer das controlirende, in dem es nicht unter die Einwirkung eines Medikaments gestellt wurde. Die Ablesungen wurden bei jeder Beobachtung dreimal wiederholt, die Zahl für den Krümmungsradius an einer für das Ophthalmometer ausgerechneten Tabelle abgelesen. Ein Schema ist folgendes: 
6. ํ̈̈r 1885.

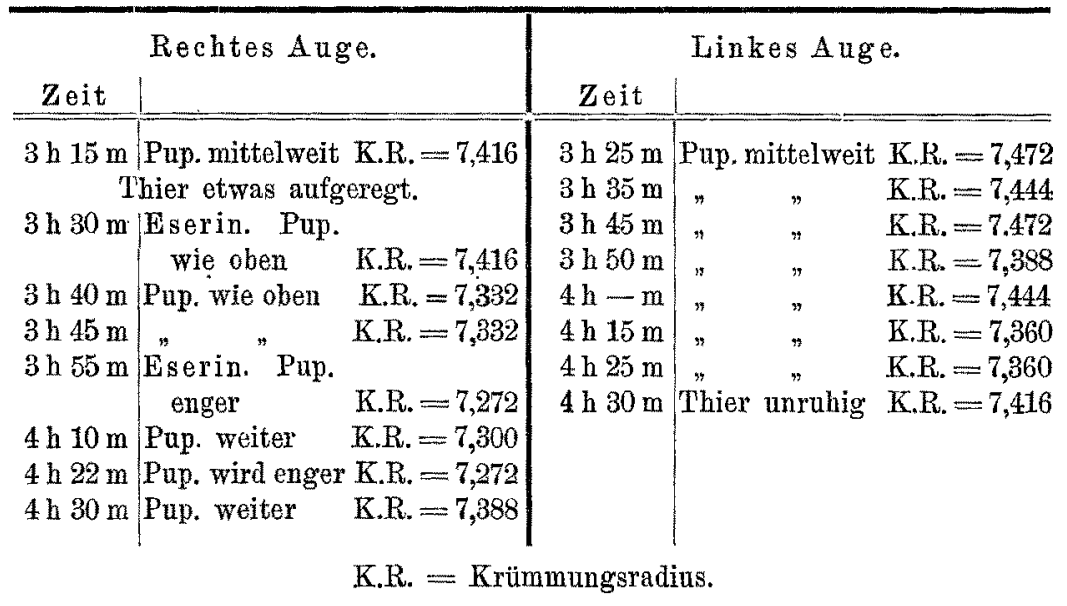

Ich habe sämmtliche vier Alcaloide, Atropin, Cocain, Eserin und Pilocarpin, auf ibren Einfluss auf die Hornhautkrümmung geprüft und kann darüber Folgendes sagen:

I. Beide Mydriatica, Atropin und Cocaïn, haben den Krümmungsradins gar nicht beeinflusst, soweit dies mit dem Ophthalmometer beurtheilt werden kann. Die Unterschiede der Ablesungen liegen innerhalb der Grenzen der Beobachtungsfehler.

II. Beide Myotica, Eserin und Pilocarpin, haben den Radius während der myotischen Wirkung stets kürzer gemacht, und zwar um 0,1 bis 0,2 Millimeter.

Mit der Beobachtung II. befinden wir uns also mit v. Reuss in Debereinstimmung, der bei Eserin stets eine Radiusverkürzang gesehen hat, und es dürfte dessen Vermuthung, den Ciliarmuskel betreffend, (siehe oben), ihre Richtigkeit haben.

Die Methode bedarf jedenfalls noch der Vervollkommnung und erst, wenn am gleichen Auge Manometer und Ophthalmometer mit einander arbeiten, dürfte in dieser Beziehung - welcher Druckhöhe eine gewisse Hornhaut- 
radiuslänge entspreche - Definitives zu Tage geî́rdert werden.

Schliesslich fühle ich mich gedrungen, meinem hochverehrten Lehrer, Herrn Prof. Dr. Pflüger und dem hochverehrten Herm Prof, Dr. Kronecker für ihre anregenden Rathschlige und thatsächliche Mithulfe bei vorliegender Arbeit meinen herzlichsten Dank auszusprechen.

Im Oktober 1885. 